\title{
The structure of elliptical galaxies in the Virgo cluster. Results from the INT Wide Field Survey ${ }^{\star}$
}

\author{
G. Gavazzi ${ }^{1}$, A. Donati ${ }^{1}$, O. Cucciati ${ }^{1}$, S. Sabatini ${ }^{2}$, A. Boselli ${ }^{3}$, J. Davies ${ }^{2}$, and S. Zibetti ${ }^{4}$ \\ 1 Universitá degli Studi di Milano-Bicocca, P.zza della Scienza 3, 20126 Milano, Italy \\ e-mail: [Giuseppe.Gavazzi ; alessandro.donati]@mib.infn.it \\ 2 Department of Physics and Astronomy, Cardiff University, UK \\ e-mail: sabina.sabatini@astro.cf.ac.uk \\ ${ }^{3}$ Laboratoire d'Astrophysique de Marseille, BP8, Traverse du Siphon, 13376 Marseille, France \\ e-mail: alessandro.boselli@oamp.fr \\ ${ }^{4}$ Max-Planck-Institut für Astrophysik, Garching, Germany \\ e-mail: zibetti@mpa-garching.mpg.de
}

Received 24 October 2003 / Accepted 16 September 2004

\begin{abstract}
We report on a complete CCD imaging survey of 226 elliptical galaxies in the North-East quadrant of the Virgo cluster, representative of the properties of giant and dwarf elliptical galaxies in this cluster. We fit their radial light profiles with the Sersic $r^{1 / n}$ model of light distribution. We confirm the result of Graham \& Guzman (2003, AJ, 125, 2936) that the apparent dichotomy between $\mathrm{E}$ and $\mathrm{dE}$ galaxies in the luminosity- $\langle\mu\rangle_{\mathrm{e}}$ plane no longer appears when other structural parameters are considered and can be entirely attributed to the onset of "core" galaxies at $B_{T} \sim-20.5$ mag. When "core" galaxies are not considered, $\mathrm{E}$ and $\mathrm{dE}$ form a unique family with $n$ linearly increasing with the luminosity.

For 90 galaxies we analyze the $B-I$ color indices, both in the nuclear and in the outer regions. Both indices are bluer toward fainter luminosities. We find also that the outer color gradients do not show any significant correlation with the luminosity. The scatter in all color indicators increases significantly toward lower luminosities, e.g. galaxies fainter than $B_{T} \sim-15$ have a $B-I$ spread $>0.5$ mag.
\end{abstract}

Key words. galaxies: elliptical and lenticular, $\mathrm{cD}$ - galaxies: fundamental parameters - galaxies: clusters: individual: Virgo

\section{Introduction}

The widespread belief that dwarf (dE) and giant (E) elliptical galaxies form two distinct families brought unanimous consent that separate mechanisms are responsible for their formation and evolution (see Ferguson \& Binggeli 1994, for a review). Alleged evidence for a structural $\mathrm{dE} / \mathrm{E}$ dichotomy includes: i) the light profiles of dEs follow exponential laws (Binggeli et al. 1984), as opposed to their giant counterparts that follow the $r^{1 / 4}$ law (de Vaucouleurs 1948); ii) scaling relations of the structural parameters $r_{\mathrm{e}}$, the effective radius, and $\langle\mu\rangle_{\mathrm{e}}$, the mean surface brightness within $r_{\mathrm{e}}$, with the total luminosity indicate that dEs of increasing luminosity have greater surface brightness while giant Es show the reverse trend (Binggeli \& Cameron 1991; Binggeli et al. 1984). Moreover they occupy distinct loci in the $r_{\mathrm{e}}$ vs. $\langle\mu\rangle_{\mathrm{e}}$ plane: giants have dimmer surface brightness with increasing radius (Kormendy \& Djorgowski 1989), while dEs do not.

Hydrodynamical simulations, including mass loss from stars and gas heating and cooling, show that gravitational

^ Table 2, Figs. 13 and 14 are only available in electronic form at http://www. edpsciences.org instability of primeval density fluctuations (with or without dark matter) results in the formation of elliptical galaxies with brighter $\langle\mu\rangle_{\mathrm{e}}$ at high luminosity, as observed in the $\mathrm{dE}$ regime, without showing any dichotomy, i.e. the bright objects result as scaled-up versions of the less luminous systems (Athanassoula 1993).

Various mechanisms involving merging have been proposed for the formation of E galaxies that are consistent with the alleged $\mathrm{dE} / \mathrm{E}$ dichotomy. Dwarf Es in clusters might derive from the harassment of dwarf spiral galaxies (Moore et al. 1996, 1998; Mao \& Mo 1998; Mayer et al. 2001), while giant E galaxies might arise from the merger of two spiral galaxies or from the multiple merging of dEs (Kauffmann et al. 1993).

There is however some observational evidence arguing for a continuity rather than a dichotomy between $\mathrm{dEs}$ and Es: i) the $U-V$ (Caldwell 1983) and $B-H$ (Scodeggio et al. 2002) colors and the metallicity are found to smoothly increase with luminosity; ii) the Near-IR light concentration index (Scodeggio et al. 2002) and the central surface brightness (Caldwell \& Bothun 1987; Jerjen \& Binggeli 1997) increase monotonously with the absolute magnitude; iii) no clear 
dichotomy can be assessed between giants and dwarfs in the Near-IR $\langle\mu\rangle_{\mathrm{e}}-R_{\mathrm{e}}$ and fundamental plane relations (Zibetti et al. 2002). Whether there is kinematic evidence for the $\mathrm{dE} / \mathrm{E}$ dichotomy remains a controversial issue: the preliminary conclusion of Geha et al. (2001) that $\mathrm{dE}$ and $\mathrm{E}$ are both not rotationsupported systems has been subsequently questioned by the same authors (Geha et al. 2003) who concluded, in agreement with Pedraz et al. (2002) and van Zee et al. (2004), that dE galaxies can have significant rotation.

Moreover the very existence of the aforementioned $\mathrm{dE} / \mathrm{E}$ dichotomy has been recently questioned by Graham \& Guzman (2003; GG03 hereafter). These authors argued that the dichotomy is apparent, as it derives from the use of exponential and de Vaucouleurs laws to decompose the light profiles of $\mathrm{dE}$ and $\mathrm{E}$ galaxies respectively, combined with the use of $\langle\mu\rangle_{\mathrm{e}}$ as representative of the surface brightness. When $\mu_{0}$ is analyzed, as obtained from the inner extrapolation of Sersic $r^{1 / n}$ laws (Sersic 1968), no dichotomy remains.

Stimulated by the conclusions of GG03 we undertook this project aimed at re-assessing the relations involving the size, the surface brightness and the luminosity of dE-E galaxies, all ingredients entering in the determination of the fundamental plane. The present analysis is carried out using a sample which by selection should be representative of the properties of $\mathrm{dE}-\mathrm{E}$ galaxies in one cluster.

The Virgo cluster, owing to its relatively small distance from us $(17 \mathrm{Mpc})$, represents an appropriate testbed for such a study, although its significant line-of-sight depth (Gavazzi et al. 1999; Solanes et al. 2002) and sub-clustering makes it not ideal. In spite of their low surface brightness, dwarf galaxies as faint as 19 mag are within reach of mid-size telescopes and seeing limitations are less severe when studying galaxies at the distance of Virgo than objects 5 times further away such as the Coma galaxies that require the superior resolution of HST. Since their luminosity function was determined (e.g. Sandage et al. 1985), early systematic studies of dwarf galaxies in Virgo were based on photographic material (Binggeli \& Cameron 1991, 1993; Young \& Currie 1998). Modern photometry obtained with panoramic detectors exists today for many Virgo galaxies (e.g. Caon et al. 1993; Gavazzi et al. 2003; Barazza et al. 2003) and many have been observed with the HST (e.g. Faber et al. 1997; Cote et al. 2004). A large stretch of the Virgo cluster was mosaiced with the Isaac Newton Telescope in what is known as the "Virgo Wide Field Survey" (Sabatini et al. 2003). These data are used in the present analysis to re-assess the issue of the $\mathrm{dE} / \mathrm{E}$ dichotomy.

The analyzed sample is described in some detail in Sect. 2. Sections 3 and 4 describe the method used to derive the light profiles and their fitting with Sersic models. The $B-I$ color analysis and the analysis of the various structural $B$-band parameters are carried out in Sect. 5 and the conclusions of our work are briefly summarized in Sect. 6.

\section{The sample}

The present structural analysis of elliptical galaxies is focused on the North-East quadrant of the Virgo cluster delimited by $\alpha>12^{\mathrm{h}} 20^{\mathrm{m}} 00^{\mathrm{s}}$ and $\delta>10^{\circ} 00^{\prime} 00^{\prime \prime}$ (see Fig. 1). With this choice we try to include in the analysis genuine members of cluster A (M 87) and to exclude members of other dynamical units within the Virgo cluster, namely cluster B (M 49), clouds $\mathrm{M}$ and $\mathrm{W}$ which are located to the West and South of the above region, showing significant 3-D structure, i.e. distances in excess of 6 (cluster B) and $15 \mathrm{Mpc}$ (M, W clouds) with respect to cluster A that we assume at $17 \mathrm{Mpc}$ distance (Gavazzi et al. 1999).

Our structural analysis requires the availability of imaging material (CCD images) suitable for deriving the light profiles according to the method described in Sect. 4. The imaging data are taken from the Wide Field Survey (WFS), horizontal (WFH) and vertical (WFV) strips delimited by the solid rectangles in Fig. 1. B-band data are available for both strips. Moreover in the vertical strip we had access to $I$-band data to study the $B-I$ color of galaxies in this region (see Sect. 5.2). WFS images are in principle available for all (439) $m_{\mathrm{p}} \leq 20$ galaxies in the Virgo Cluster Catalog (VCC; Binggeli et al. 1985) projected onto the WFS region ${ }^{1}$. We restricted however the present analysis to the 251 galaxies with $m_{\mathrm{p}} \leq 19$, with morphological type $\mathrm{dE}$ or $\mathrm{E}$, that are spectroscopically confirmed members $\left(V<3000 \mathrm{~km} \mathrm{~s}^{-1}\right)$ or possible members according to Binggeli et al. (1985, 1993). We were able to fit meaningful B Sersic profiles to 226 galaxies out of 251 because 18 were not observed and the remaining 7 were too faint or the images were of insufficient quality. Summarizing, $B$ band structural information is available for 226/251 objects, thus with a completeness of $90 \%$. The $I$-band structural information is available for 90 objects. The sample completeness is detailed in Table 1. At the assumed distance of $17 \mathrm{Mpc}$ $(\mu=-31.15)$, the limit of $m_{\mathrm{p}}=19 \mathrm{mag}$ corresponds to $M_{\mathrm{p}}=-12.15$. Therefore the analyzed sample can be considered representative of the properties of $\mathrm{dE}-\mathrm{E}$ galaxies in the $\mathrm{N}-\mathrm{W}$ portion of the Virgo cluster. Figure 2 illustrates the luminosity function of dE-E galaxies included in the present analysis. It is in full agreement with the one derived by Sandage et al. (1985, see their Fig. 11) for the entire VCC, once re-normalized to account for the $28 \%$ coverage of the WFS with respect to the entire cluster and for the different distance modulus assumed.

\section{The imaging data}

The Virgo Wide Field Survey was carried out with the Wide Field Camera (WFC) at the Isaac Newton Telescope (INT) (Sabatini et al. 2003).

The horizontal strip was covered with 55 overlapping fields of $34 \times 22$ arcmin each, using 3 of the 4 chips of $4000 \times 2000$ pixel (each of 0.333 arcsec on the sky)

\footnotetext{
${ }^{1}$ Nine VCC galaxies in the WFH (VCC 1143, 1147, 1216, 1335, $1336,1482,1486,1795,1886)$ and nine in the $\operatorname{WFV}(281,333,400$, $437,723,742,769,863,884$ ) were not observed in the $B$-band because they lie in the gaps between adjacent chips of the WFC; similarly 15 objects in the WFV (I-band) (VCC 400, 413, 505, 578, 663, 666, 677, 723, 751, 769, 797. 798. 857, 882, 884).
} 


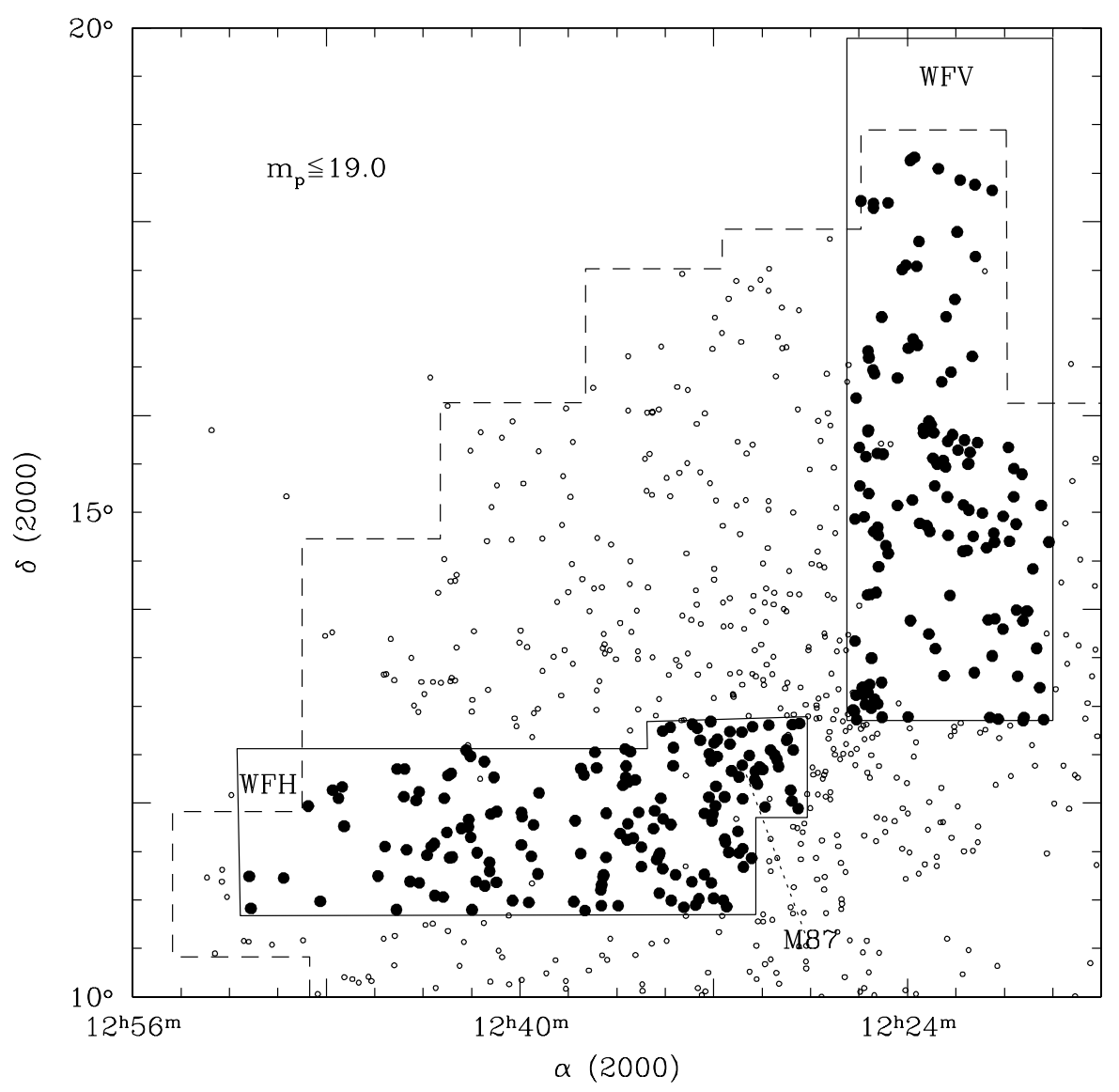

Fig. 1. The N-E region of the Virgo cluster including galaxies with $m_{\mathrm{p}} \leq 19.0$. The dashed line represents the boundary of the VCC catalog. The rectangles include the studied WFH and WFV areas, covering approximately $26 \mathrm{deg}^{2}$. The location of M 87 is indicated.

constituting the $\mathrm{WFC}^{2}$. For this strip we had access only to the $B$-band (Johnson) data. The observations were carried out in nearly photometric conditions with a seeing varying from 1 to 3 arcsec, with a mean of 1.6 arcsec (see Fig. 3). An integration time of $750 \mathrm{~s}$ was used.

The Vertical strip was covered in both the $B$ - and the $I$-band. The $B$-band survey was carried out with 80 overlapping fields, using 3 of the 4 chips of the WFC, each exposed for $750 \mathrm{~s}$. The observations were taken in nearly photometric conditions with a seeing varying from 1.2 to 3.5 arcsec, with a mean of 2.1 arcsec.

The $I$-band survey consists of 73 overlapping fields ${ }^{3}$, each exposed for $1000 \mathrm{~s}$. The observations were carried out in nearly photometric conditions with a seeing varying from 1 to 2.5 arcsec, with a mean of 1.3 arcsec.

\section{Data reduction}

The reduction of the $B$-band science frames was performed as described in Sabatini et al. (2003). The zero

\footnotetext{
2 The WFC is made of 3 chips, one on top of the other, and of one orthogonal chip on one side. The top horizontal chip is partly vignetted. This chip was not used in the $B$-band survey, but it was considered in the $I$-band (see next section).

3 The 7 missing $I$-band frames lie at $\delta>18^{\circ} 00^{\prime}$, in a region almost devoid of VCC galaxies.
}

Table 1. The sample. For each band the number of dE-E galaxies in the WFS (separately for the Horizontal and Vertical strips) with $m_{\mathrm{p}} \leq$ 19 mag. that are members or possible members, to which we have been able to fit a Sersic profile is given. The denominator gives the number of galaxies in the VCC at the same magnitude limit.

\begin{tabular}{ccc}
\hline \hline & $B$ & $I$ \\
Strip & $m_{\mathrm{p}} \leq 19$ & $m_{\mathrm{p}} \leq 19$ \\
\hline WFH & $131 / 149(88 \%)$ & - \\
WFV & $95 / 102(93 \%)$ & $90 / 102(89 \%)$ \\
\hline
\end{tabular}

point of all images was obtained from the "CASU INT Wide Field Survey Home Page" (http://www.ast.cam. ac. uk/ wfcsur/index.php).

The I-band images suffer from fringing, producing unwanted structures in the background. However for this band we could access images produced by all 4 chips of the WFC, including the top, redundant, vignetted chip. Several galaxies were observed in non-vignetted parts of this chip as well as in other chips of other frames. In these cases we combined two independent sets of measurements to reduce the fringing.

Because of the significantly better seeing in the $I$ - compared to the $B$-band, the $I$-band profile decomposition (see next section) was performed twice: once on the original data, 


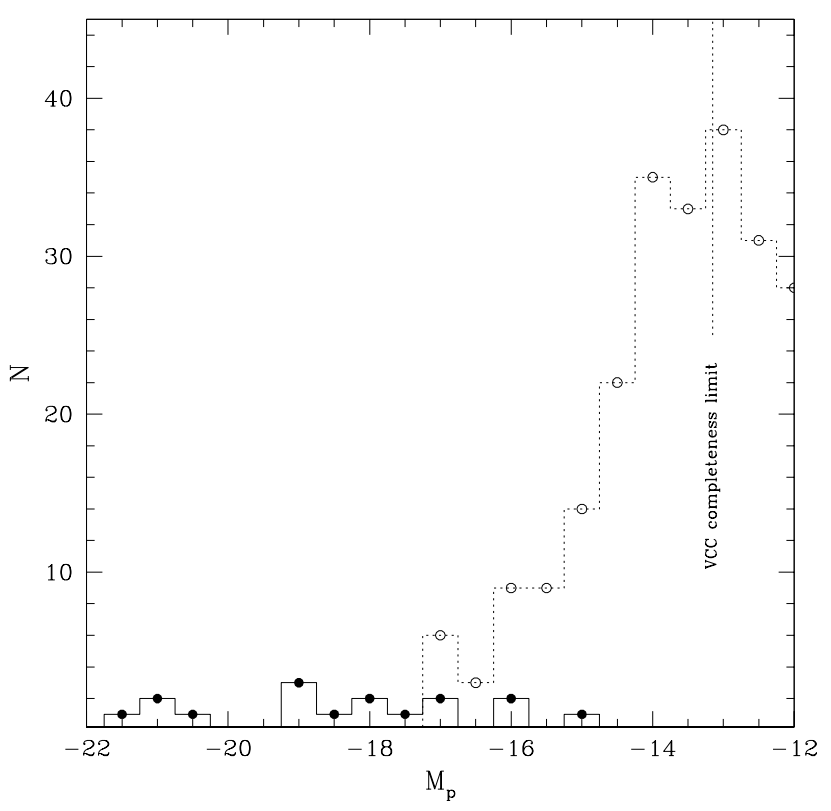

Fig. 2. The luminosity function of E (filled symbols) and dE (open symbols $)$ in the WFS. The completeness limit of the VCC $\left(m_{\mathrm{p}}=18\right)$ is indicated.
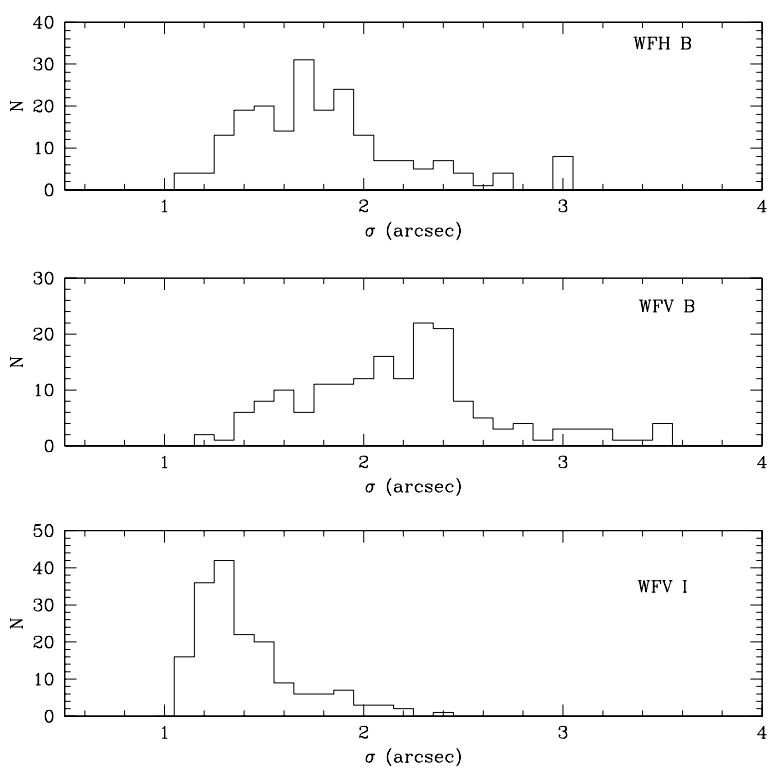

Fig. 3. Distribution of the seeing in the WFS.

once on degraded images obtained convolving the images to the $B$-band seeing.

\subsection{Profile decomposition}

The profile decomposition is performed using the method described in Gavazzi et al. (2000, 2001) which is based on the IRAF environment and relies on the STSDAS ${ }^{4}$ package and

${ }^{4}$ IRAF is the Image Analysis and Reduction Facility made available to the astronomical community by the National Optical Astronomy Observatories, which are operated by AURA, Inc., under contract with the US National Science Foundation. STSDAS is distributed by the Space Telescope Science Institute, which is operated by on GALPHOT (developed for IRAF- STSDAS mainly by W. Freudling, J. Salzer, and M.P. Haynes and adapted by M. Scodeggio, P. Franzetti and S. Zibetti).

For each frame the sky background is determined as the mean number of counts measured in regions of "empty" sky, and it is subtracted from the frame.

The 2-dimensional light distribution of each galaxy is fitted with elliptical isophotes, using a procedure based on the task ellipse, (STSDAS ISOPHOTE package; Jedrzejewski 1987; Busko 1996), which allows the interactive masking of unwanted superposed stars and galaxies. Starting from an interactively centered ellipse, the fit maintains as free parameters the ellipse center, ellipticity and position angle. The ellipse semimajor axis is incremented by a fixed fraction of its value at each step of the fitting procedure. The routine halts when the surface brightness found in a given annulus equals the the average sky value, and then restarts decrementing the initial semi-major axis toward the center. The fit fails to converge for some very faint galaxies. In these cases we keep fixed one or more of the ellipse parameters.

The resulting surface brightness profiles are fitted using the Sersic model (Sersic 1968) of light distribution:

$I(r)=I_{0} \mathrm{e}^{-\left(r / r_{0}\right)^{1 / n}}$

where $I$ is the surface brightness (in intensity) at the radius $r$ (along the major axis), $I_{0}$ is the central surface brightness, $r_{0}$ the scale length and $n$ is the dimensionless shape parameter that determines the curvature of the profile. This model is a simple generalization of de Vaucouleurs $(n=4)$ and exponential $(n=1)$ law. The surface brightness profiles are fitted with this model in the magnitude representation:

$\mu(r)=\mu_{0}+1.086\left(r / r_{0}\right)^{1 / n}$

where $\mu(r)$ is the surface brightness (in magnitude) at the radius $r$ (along the major axis), $\mu_{0}$ is the central surface brightness.

The fit is performed using a weighted least squares method from a radius equal to one seeing disk $(\sigma)$ (with the exceptions discussed in Sect. 4.2), out to the outermost significant isophotes, i.e. when the surface brightness equals the average sky value. We deliberately avoid fitting the nuclear features.

The total asymptotic magnitude $B_{T}=-2.5 \log \left(F_{T}\right)$ is obtained by adding to the flux measured $\left(F_{\text {last }}\right)$ within the outermost significant isophote $\left(r_{\text {last }}\right)$ the extra flux extrapolated to infinity along the model:

$F_{\text {extra }}=2 \pi I_{0}(1-\epsilon) n r_{0}^{2}\left[\Gamma(2 n)-\gamma\left(2 n,\left(\frac{r_{\text {last }}}{r_{0}}\right)^{1 / n}\right)\right]$

where $\epsilon$ is the ellipse eccentricity, $\Gamma$ and $\gamma$ are the complete and incomplete Gamma functions respectively. The effective radius $r_{\mathrm{e}}$ (the radius containing half of the total light) and the effective surface brightness $\langle\mu\rangle_{\mathrm{e}}$ (the mean surface brightness within $r_{\mathrm{e}}$ ), $r_{25}, r_{75}$ (the radii that enclose $25 \%$ and $75 \%$ of the total light) of each galaxy are computed empirically

the Association of Universities for Research in Astronomy (AURA), Inc., under NASA contract NAS 5-26555. 

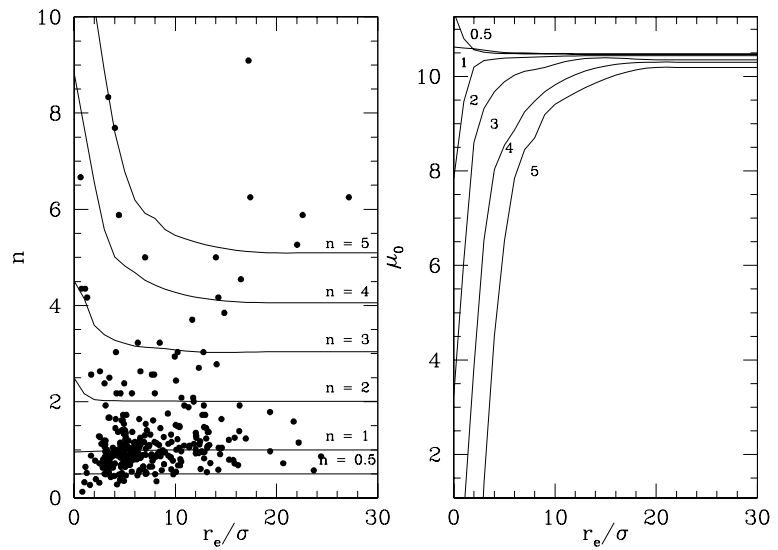

Fig. 4. The effect of the seeing on $n$ (left $)$ and on $\mu_{0}$ (right) as derived from the simulations (lines) for various $n$. Dots give the distribution of the observed points in the $n-r_{\mathrm{e}} / \sigma$ plane.

from $B_{T}$, i.e. are derived from the data, using the Sersic models to extrapolate from the last measured magnitude to infinity. Similarly we compute the concentration index $\left(C_{31}\right)$, defined in de Vaucouleurs (1977) as the ratio between $r_{75}$ and $r_{25}$. Fits to the $B$-band light profiles were obtained for 136 galaxies, as given in Fig. 13, and to $B+I$-band light profiles for 90 objects, as given in Fig. 14.

\subsection{The effects of the seeing}

Attempts to model the effects of the seeing on Sersic parameters can be found in the literature, e.g. by Trujillo et al. (2001a,b) who modeled the effect of Gaussian and Moffat convolution on Sersic profiles. To illustrate this issue we used some simple simulations. We constructed sets of artificial images injecting fake Sersic galaxies with zero ellipticity, constant $\mu_{0}$, fixed $r_{\mathrm{e}}=10 \operatorname{arcsec}$ and Sersic index $n$ varying from 0.5 to 5 on a sky background with noise characteristics similar to the INT frames. To simulate the effects of the seeing the images were convolved with Gaussians of $F W H M(\sigma)$ ranging from 0.5 to 10 arcsec, so that $r_{\mathrm{e}} / \sigma$ varies between 20 and 1 , mimicking the range covered by the real data. We fitted circular isophotes and measured the Sersic parameters on the blurred images with the same tools and criteria used for real data (i.e. disregarding the data points within the seeing disk). Figure 4 illustrates how the measured Sersic parameters $n$ and $\mu_{0}$ vary as a function of $r_{\mathrm{e}} / \sigma$ (lines). Dots give the observed distribution in the $n-r_{\mathrm{e}} / \sigma$ plane. From Fig. 4 it is apparent that increasing corrections to the Sersic parameters are required with increasing $n$ and decreasing $r_{\mathrm{e}} / \sigma$. For $n \geq 3$ the seeing produces overestimates of $n$ (thus enhancing the central surface brightness $\mu_{0}$ ) because flux from the central cusp is distributed in the surrounding pixels producing a steepening of the inner profile.

As a result of the simulations we decided not to try to correct the individual Sersic parameters (that are not independent of one another), but to modify our fitting strategy in the case of $n \geq 3$, i.e. excluding from the fit the data points within $2 \times$ the seeing disk $\sigma$.

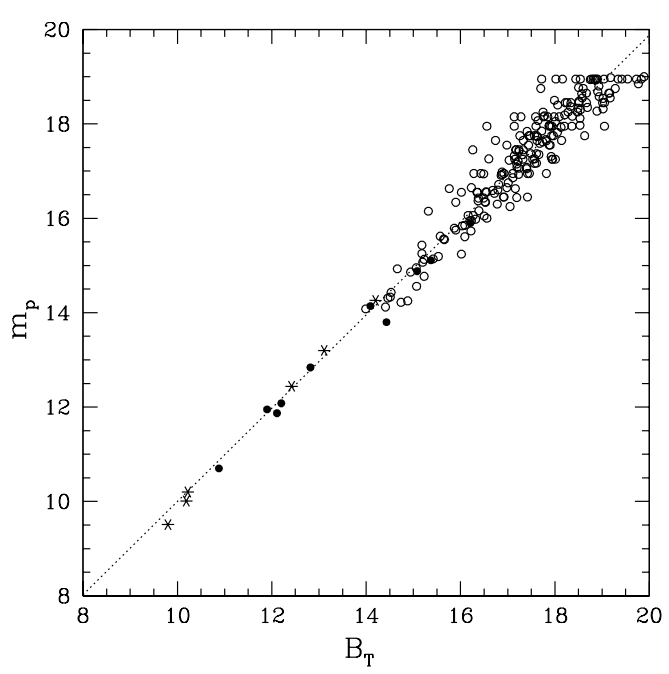

Fig. 5. Comparison of $B_{T}$ from this work with $m_{\mathrm{p}}$ from the VCC. The dotted line gives the bisector linear regression. In this and in the following figures (unless otherwhise specified) empty symbols refer to dEs, filled symbols to Es and asterisks to "core" galaxies.

\section{Results}

The $B$-band parameters derived in this work are listed in Table 2. Magnitudes and surface brightness are corrected for extinction in our Galaxy according to Burstein \& Heiles (1982) (due to the high galactic latitude of the Virgo cluster this results in a small 0.05 mag correction on average included in Cols. 4, 6,7 , and 9).

\subsection{Consistency test}

The quality of the measurements in Table 2 is first checked by comparing the total asymptotic magnitudes $\left(B_{T}\right)$ derived in this work with $m_{\mathrm{p}}$ given in the VCC (see Fig. 5). $B_{T}$ is found in linear proportionality with $m_{\mathrm{p}}$. The bisector linear regression (see Feigelson \& Babu 1992) is:

$m_{\mathrm{p}}=+0.11+0.99 B_{T}(R=0.96)$

where $R$ is the Pearson regression coefficient.

Figure 6 illustrates the comparison between the parameters derived in this work on CCD frames with the parameters derived on photographic material by Young \& Currie (1998, VPC) for the 42 common galaxies (left) and by Binggeli \& Jerjen (1998) (based on photographic data of Binggeli \& Cameron 1991) for the 37 common galaxies (right). The agreement between the two sets of measurements is satisfactory, except for some deviating objects marked individually in the figure with their VCC denominations. The three most discrepant objects (i.e. VCC $748,833,1565$ ) have $B_{T}$ derived in this work consistent within 0.28 mag with $m_{\mathrm{p}}$ from the VCC, differing by more than $1.5 \mathrm{mag}$ from the VPC values. Furthermore we checked the $n$ index on the profiles of VCC 748, 1148 and 1308 and confirmed that the value found in this work is correct.

We remark that 18 galaxies are in common between this work, Young \& Currie (1998) and Binggeli \& Jerjen (1998). For these objects we compare the Sersic parameters on 3 independent data-sets and we conclude that the consistency is best 

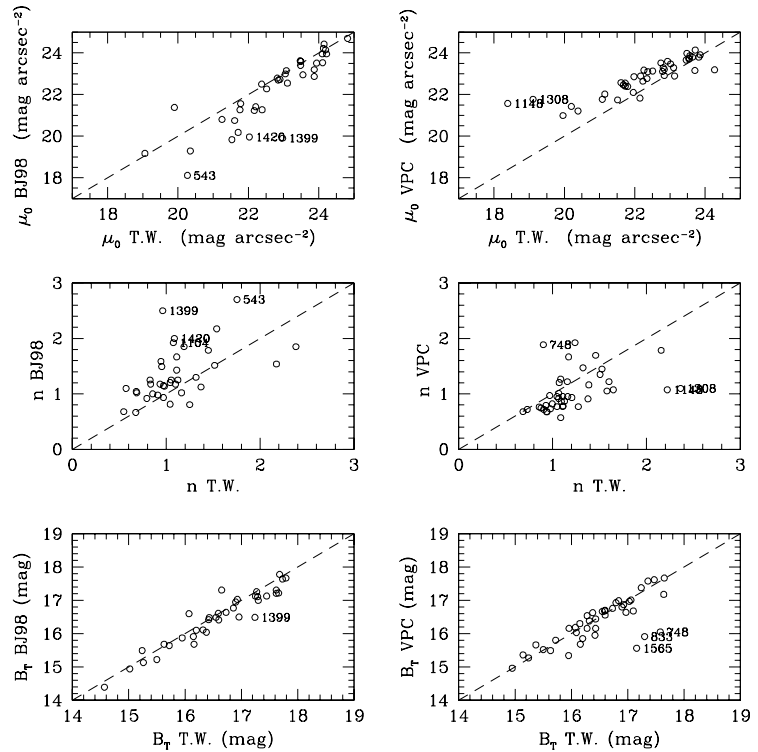

Fig. 6. Comparison among structural parameters derived in this work and by Young \& Currie (1998) for 42 common galaxies (right) and by Binggeli \& Jerijn (1998) for 37 common galaxies (left).
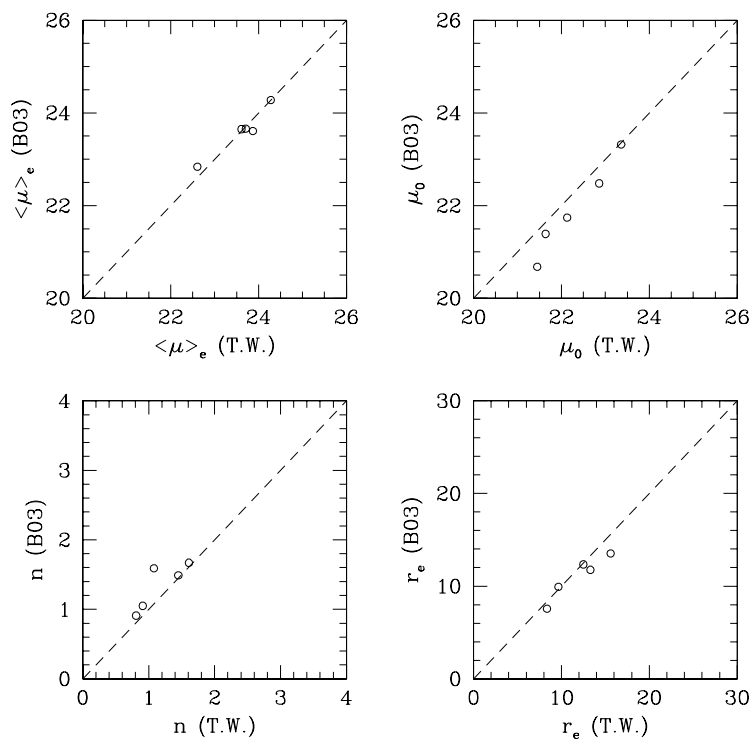

Fig. 7. Comparison among structural parameters derived in this work and by Barazza et al. (2003) for 5 common galaxies.

between this work and the VPC and worst between Young \& Currie and Binggeli \& Jerjen (1998). In other words the average errors in the VPC are approx. 1.5 times larger than those in this work while those of Binggeli \& Jerjen (1998) are approx. 3 times larger than those in this work.

Indeed when we compare the results of this work with those obtained by Barazza et al. (2003) on CCD material for the 5 common galaxies (see Fig. 7) we find a high consistency.

\subsection{B-I color analysis}

Using 90 galaxies in the WFV that have been observed both in the $B$ and in the $I$-band we analyze in this section the $B-I$ color properties of dE-E galaxies. For these objects the $B$ band Sersic
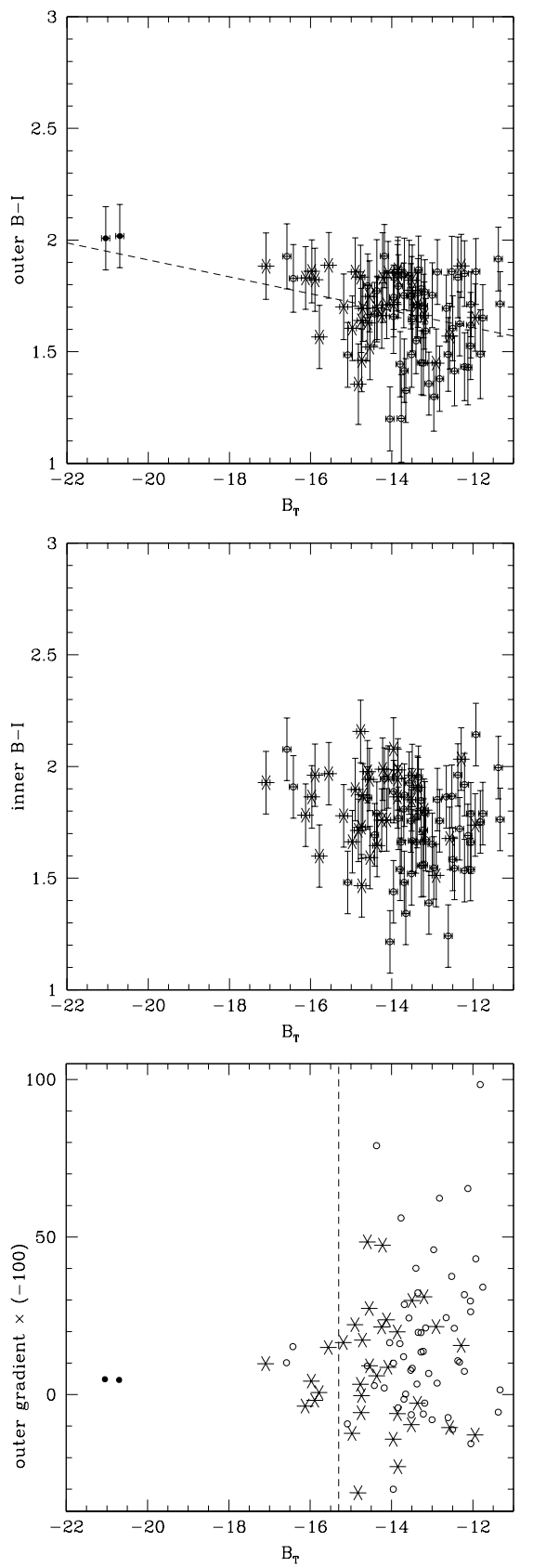

Fig. 8. The outer $B-I$ color index (top); the inner $B-I$ color index (middle; the two brightest objects are missing in this panel because they are both saturated) and the outer $B-I$ gradient (bottom) as a function of $B_{T}$. In this figure filled dots represent giant Es, empty dots dEs, asterisks Nucleated dEs.

parameters were compared with two sets of similar $I$ band parameters, i.e. those derived from the original $I$ images and from the $I$ images convolved to the $B$-band seeing. The agreement between the $B$ and $I$ parameters is satisfactory and it improves when the $B$ parameters are compared with those obtained from the blurred $I$ images.

For these objects we also compute the outer $B-I$ color index in the radial interval between $r>1.5 \times \sigma$ and (two points before) the last significant measurement, as representative of the galaxy color outside the nucleus, avoiding the least reliable, outermost points. Figure 8 (top) gives the color-magnitude 
diagram obtained in this way, showing the expected trend of blueing toward fainter luminosities (see Baldry et al. 2004). We remark however that our WFV data-set contains too few giant E galaxies to explore the relation at high luminosity. It appears that, fainter than $B_{T}=-16$, the scatter in the relation becames increasingly large.

For the same set of galaxies we also compute the "outer color gradient" as the slope of the line fitted to the $B-I$ color profile in the radial interval between $r>1.5 \times \sigma$ and (two points before) the last significant measurement. When plotting the outer gradient we follow the convention of Vader et al. (1988) of "positive" gradients when the the center is redder. Figure 8 (bottom) shows the outer gradients as a function of the B luminosity, revealing no significant correlation. We do not confirm the trend found by Vader et al. (1988) in a brighter $\left(B_{T}<-15.3\right.$, vertical line) sample who claimed that brighter galaxies have more positive gradients (outer envelopes bluer than inner regions) than fainter objects. Our fainter sample shows that this trend does not hold at faint luminosities where the outer gradients show a tremendous scatter.

Figure 8 (middle) shows the inner (or nuclear) color index obtained as the difference of the $B$ and $I$ magnitudes integrated within $1 \times \sigma$. There is only a weak tendency for redder nuclei in brighter objects, but again with a large uncertainty.

\subsection{Correlations among $B$-band structural parameters}

Guided by the work of GG03 who based their study on a sample of $18 \mathrm{dEs}$ in Coma observed with the HST, combined with 232 elliptical galaxies taken from the literature, we analyze in this Section several correlations among structural parameters of dE-E galaxies in the Virgo cluster that are derived using the Sersic model.

We remark that the sample of Virgo elliptical galaxies we are using is $90 \%$ complete at $m_{\mathrm{p}}<19$. In other words the density of points in the various plots reflects the real frequency in the parameter space of Virgo galaxies.

We begin by remarking, in full agreement with GG03 and Caon et al. (1993), that in our sample there is a significant linear increase of the Sersic index $n$ with the system luminosity (see Fig. 9). Using the bisector linear regression we find:

$\log (n)=-0.12 \times B_{T}+1.71(R=-0.72)$.

All dEs with $B_{T}>-17$ have Sersic index $n \lesssim 2$ while the few giant Es have $n$ as high as 7 .

Secondly we show in Fig. 10 (top) that the relation $B_{T}$ versus $\langle\mu\rangle_{\mathrm{e}}$, extensively studied by Binggeli \& Cameron (1991), Binggeli et al. (1984) and Ferguson \& Binggeli (1994) shows the existence of two separate regimes: dwarf elliptical galaxies having brighter surface brightness with increasing luminosity, and giants showing the reverse trend. Furthermore the relation between the effective surface brightness and the radius (see Fig. 10, middle) is of inverse proportionality for giant ellipticals (Kormendy \& Djorgowski 1989), i.e. smaller radii at brighter mean surface brightness, while dEs show a sparse relation (see also Capaccioli \& Caon 1991). Third we show (bottom) that the scale $\log R_{\mathrm{e}}$ increases with $B_{T}$ more rapidly for giant Es than for dEs (see Binggeli et al. 1984).

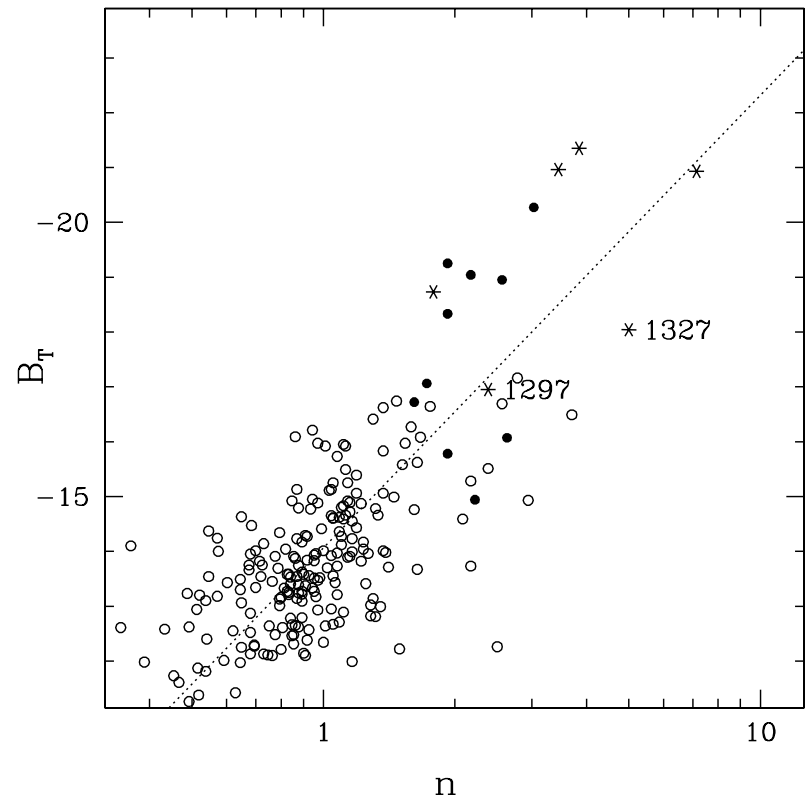

Fig. 9. The dependence of $n$ from $B_{T}$. The dashed line gives the bisector linear regression.

In all panels of Fig.10 it appears that some dichotomy occurs in passing from dEs to Es. However, as stressed for the first time by GG03, all bright galaxies showing deviant trends in this figure are the "partially evacuated core" or "core" galaxies that show a flat slope in the inner $\$ 100 \mathrm{pc}(\$ 1.2 \operatorname{arcsec}$ at the distance of Virgo), as opposed to the "normal" galaxies with a central cusp (that Faber et al. 1997, define as "power-law" galaxies). It should be noted that the region of the Virgo cluster mapped with completeness in this work does not contain many "core" galaxies, beside M 84, M 86 and M 87 (the brightest objects in Fig. 10). Most "core" objects found in other clusters by Faber et al. (1997) are much more luminous $\left(-21<B_{T}<-24\right)$ cD galaxies with large outer envelopes that are absent in Virgo. These galaxies obey Sersic laws only in the outer profiles and Graham et al. (2003b) have developed a formalism to model their profiles in their full extent. The rectangles in Fig. 10 represent the loci occupied by them. Notice that the M 32-like objects (e.g. VCC 1297 and 1327) (both core galaxies) represent the low-luminosity continuation of the core regime.

The E-dE dichotomy no longer appears when $B_{T}$ is plotted as a function of $\mu_{0}$ in Fig. 11. GG03 conclude that "normal" dE-E galaxies have increasingly brighter central surface brightness with increasing luminosity, until the onset of "core" formation in elliptical galaxies at $B_{T} \sim-20.5 \mathrm{mag}$. Together with GG03 we conclude that among "normal" E-dE galaxies there is no dichotomy in the structural parameters. Only the "core" galaxies seem structurally different, perhaps due to a different formation mechanism (see the discussion in Graham et al. 2003b).

Given the significant correlation between $B_{T}$ and $\log n$, which clearly indicates a smooth, continuous transition from 

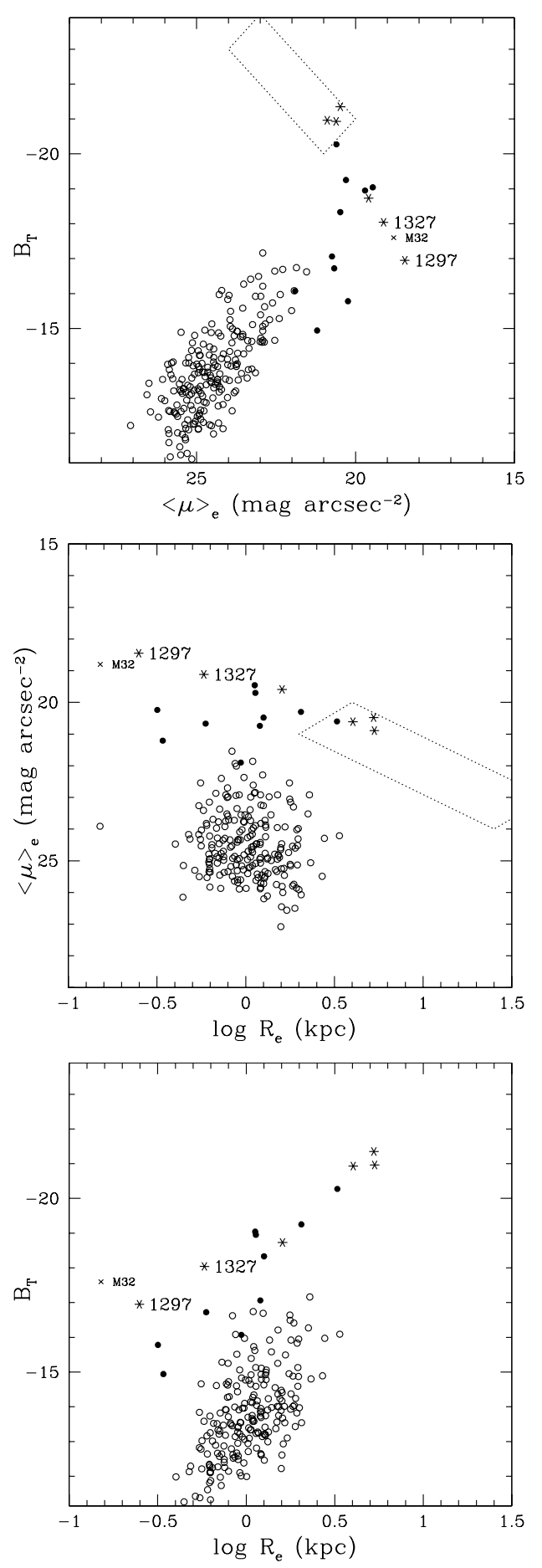

Fig. 10. Correlations among structural parameters of dE-E galaxies in the Virgo cluster. $B_{T}$ vs. $\langle\mu\rangle_{\mathrm{e}}($ top $) ;\langle\mu\rangle_{\mathrm{e}}$ vs. $\log R_{\mathrm{e}}$ (middle); $B_{T}$ vs. $\log R_{\mathrm{e}}$ (bottom). The position of M 32 (scaled to the distance of Virgo and corrected for $B-V=1$ ) as given by Faber et al. (1997) is marked with a cross. The dotted rectangle represents the loci of luminous "core" galaxies adapted from GG03.

the $\mathrm{dE}$ to the E regime, and the (analytically ${ }^{5}$ expected) correlation between the light concentration index $C_{31}$ and the Sersic

\footnotetext{
${ }^{5} C_{31}$ and $n$ are expected to have a perfect correlation in the Sersic model. However in our definition $C_{31}$ is empirically determined from the data.
}

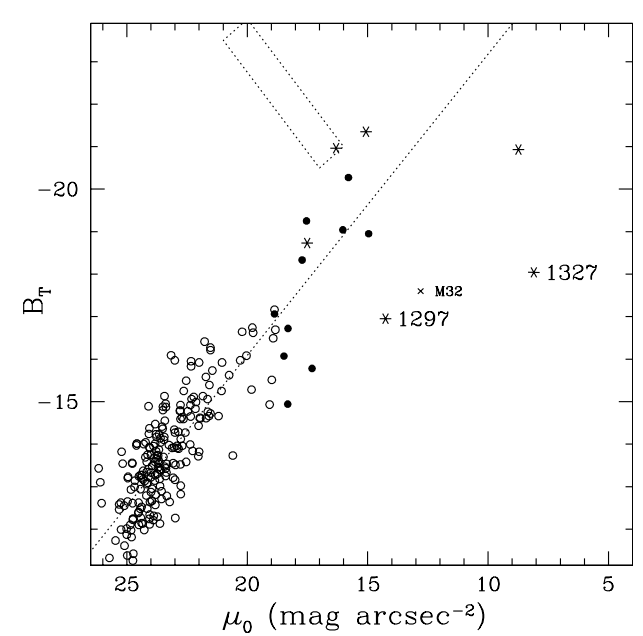

Fig. 11. Correlation between $B_{T}$ and $\mu_{0}$ for dE-E galaxies in the Virgo cluster. The position of M 32 is marked with a cross. The dotted rectangle represents the loci of luminous "core" galaxies adapted from GG03. The dashed line gives the bisector linear regression.

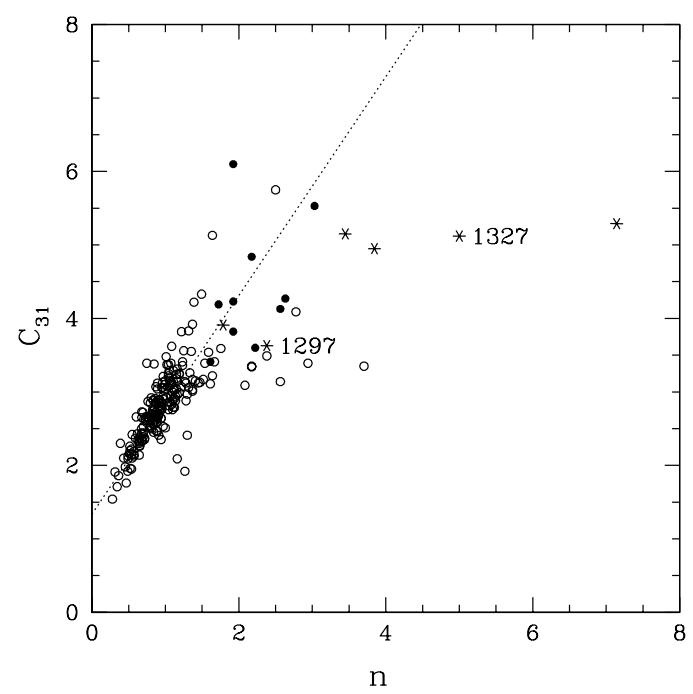

Fig. 12. Distribution of $C_{31}$ versus $n$. The dashed line gives the bisector linear regression: $C_{31}=1.49 \times n+1.33$.

index $n$ (see Fig. 12), it is not surprising that $C_{31}$ increases linearly with the total luminosity $B_{T}$. This has been known since our Near-IR survey, as stressed by Scodeggio et al. (2002).

An additional element of continuity between $\mathrm{dE}$ and $\mathrm{E}$ is the color-luminosity diagram of Fig. 8 The gradual increase of the $B-I$ index with increasing luminosity, resulting from the metallicity-mass relation (Arimoto \& Yoshii 1987), encompasses the whole $\mathrm{dE}-\mathrm{E}$ sequence, although with a larger scatter among dEs.

\section{Summary and conclusions}

We carried out a CCD survey of the North-East quadrant of the Virgo cluster containing $\sim 30 \%$ of the galaxies in this cluster. We analyzed images for 226 and 90 galaxies respectively in the $B$ - and $I$-band, representative of the properties of giant and dwarf elliptical galaxies in this cluster. 
We fit the galaxies' radial light profiles with the Sersic $r^{1 / n}$ model of light distribution obtaining the structural parameters $n,\langle\mu\rangle_{\mathrm{e}}, r_{\mathrm{e}}, B_{T}, C_{31}$ and $\mu_{0}$. We find that the Sersic model provides an adequate representation of the observed light profiles of $\mathrm{dE}$ and $\mathrm{E}$ galaxies spanning 9 mag.

We confirm the result of Graham \& Guzman (2003) that the apparent dichotomy between $\mathrm{E}$ and $\mathrm{dE}$ galaxies in the luminosity $-\langle\mu\rangle_{\mathrm{e}}$ plane is due to the onset of "core" formation in elliptical galaxies at $B_{T} \sim-20.5 \mathrm{mag}$. The segregation among $\mathrm{dE}$ and $\mathrm{E}$ no longer appears when other structural parameters are considered.

For 90 galaxies in the WFV we analyze the $B-I$ color indices, both in the nuclear and in the outer regions. Both indices are bluer toward fainter luminosities, however the scatter in these relations increases significantly toward lower luminosities. Moreover we find that the outer color gradients do not show any significant correlation with the luminosity.

Acknowledgements. We wish to thank Luca Cortese for the useful discussions. This work could not have been completed without the NASA/IPAC Extragalactic Database (NED) which is operated by the Jet Propulsion Laboratory, Caltech under contract with NASA. This research has made use also of the GOLDMine Database (http://goldmine.mib.infn.it).

\section{References}

Arimoto, N., \& Yoshii, Y. 1987, A\&A, 173, 23

Athanassoula, E. 1993, Proc. ESO/OHP Conf. on Dwarf Galaxies

Baldry, I. K., Glazebrook, K., Brinkmann, J., et al. 2004, ApJ, 600, 681

Barazza, F. D., Binggeli, B., \& Jerjen, H. 2003, A\&A, 407, 121 (B03)

Binggeli, B., Sandage, A., \& Tammann, G. 1985, AJ, 90, 1681 (VCC)

Binggeli, B., \& Cameron, L. M. 1991, A\&A, 252, 27

Binggeli, B., \& Cameron, L. M. 1993, A\&A, 98, 297

Binggeli, B., \& Jerjen, H. 1998, A\&A, 333, 17 (BJ98)

Binggeli, B., Sandage, A., \& Tarenghi, M. 1984, AJ, 89, 64

Binggeli, B., Popescu, C. C., \& Tammann, G. A. 1993, A\&AS, 98, 275

Burstein, D., \& Heiles, C. 1982, AJ, 87, 1165

Busko, I. 1996, Proc. Fifth Astronomical Data Analysis Software and Systems Conf., ed. G. H. Jacoby, \& J. Barnes, 101, Tucson PASP Conf. Ser., 139

Caldwell, N. 1983, AJ, 88, 804

Caldwell, N., \& Bothum, G. D. 1987, AJ, 94, 1126

Caon, N., Capaccioli, M., \& D’Onofrio, M. 1993, MNRAS, 265, 1013

Capaccioli, M., \& Caon, N. 1991, MNRAS, 248, 523

Cote, P., Blakeslee, J. P., Ferrarese, L., et al. 2004, ApJS, 153, 223

de Vaucouleurs, G. 1948, Annales d'Astrophysique, 11, 247 de Vaucouleurs, G. 1977, in Evolution of Galaxies and Stellar Populations, ed. R. Larson, \& B. Tinsley (New Haven: Yale University Observatory), 43

Faber, S. M., Tremaine, S., Ajhar, E., et al. 1997, AJ, 114, 1771 (F97)

Feigelson, E. D., \& Babu, G. J. 1992, ApJ, 397, 55

Ferguson, H. C., \& Binggeli, B. 1994, A\&AR, 6, 67

Gavazzi, G., Boselli, A., Scodeggio, M., Belsole, E., \& Pierini, D. 1999, MNRAS, 304, 595

Gavazzi, G., Franzetti, P., Scodeggio, M., Boselli, A., \& Pierini, D. 2000, A\&A, 361, 863

Gavazzi, G., Zibetti, S., Boselli, A., et al. 2001, A\&A, 372, 29

Gavazzi, G., Boselli, A., Donati, A., Franzetti, P., \& Scodeggio, M. 2003, A\&A, 400, 451

Geha, M., Guhathakurta, P., \& van der Marel, R. P. 2001, Am. Astr. Soc. Meeting, 199

Geha, M., Guhathakurta, P., \& van der Marel, R. P. 2003, AJ, 126, 1794

Graham, A. W., \& Guzman, R. 2003, AJ, 125, 2936 (GG03)

Graham, A. W., Erwin, P., Trujillo, I., \& Asensio Ramos, A. 2003b, AJ, 125, 2951

Jedrzejewski, R. 1987, MNRAS, 226, 747

Jerjen, H., \& Binggeli, B. 1997, in The Nature of Elliptical Galaxies, ed. M. Arnaboldi, G. S. Da Costa, \& P. Saha (San Francisco: ASP), ASP Conf. Ser., 116, 239

Kauffmann, G., White, S. D. M. \& Guiderdoni, B. 1993, MNRAS, 264, 201

Kormendy, J., \& Djorgovski, S. 1989, ARA\&A, ASP Conf. Ser., 116, 27,235

Mao, S., \& Mo, H. J. 1998, MNRAS, 296, 847

Mayer, L., Governato, F., Colpi, M., et al. 2001, ApJ, 559, 754

Moore, B., Katz, N., Lake, G., Dressler, A., \& Oemler, A., Jr. 1996, Nature, 379, 613

Moore, B., Lake, G., \& Katz, N. 1998, ApJ, 495, 139

Pedraz, S., Gorgas, J., Cardiel, N., Sánchez-Blázquez, P., \& Guzmán, R. 2002, MNRAS, 332, L59

Sabatini, S., Davies, J., Scaramella, R., et al. 2003, MNRAS, 341, 981

Sandage, A., Binggeli, B., \& Tammann, G. A. 1985, AJ, 90, 1759

Scodeggio, M., Gavazzi, G., Franzetti, P., et al. 2002, A\&A, 384, 812

Sersic, J. L. 1968, Atlas de Galaxes Australes, Cordoba Obs. Astron., Univ. Nac. Corodba

Solanes, J. M., Sanchis, T., Salvador-Solé, E., Giovanelli, R., \& Haynes, M. P. 2002, AJ, 124, 2440

Trujillo, I., Aguerri, J. A. L., Cepa, J., \& Gutiérrez, C. M. 2001a, MNRAS, 321, 269

Trujillo, I., Aguerri, J. A. L., Cepa, J., \& Gutiérrez, C. M. 2001b, MNRAS, 328, 977

Vader, J. P., Vigroux, L., Lachieze-Rey, M., \& Souviron, J. 1988, A\&A, 203, 217

van Zee, L., Skillman, E. D., \& Haynes, M. P. 2004, AJ, 128, 121

Young, C. K., \& Currie, M. J. 1998, A\&AS, 127, 367 (VPC)

Zibetti, S., Gavazzi, G., Scodeggio, M., Franzetti, P., \& Boselli, A. 2002, ApJ, 579, 261 


\section{Online Material}


G. Gavazzi et al.: INT WFS in Virgo, Online Material $p 2$
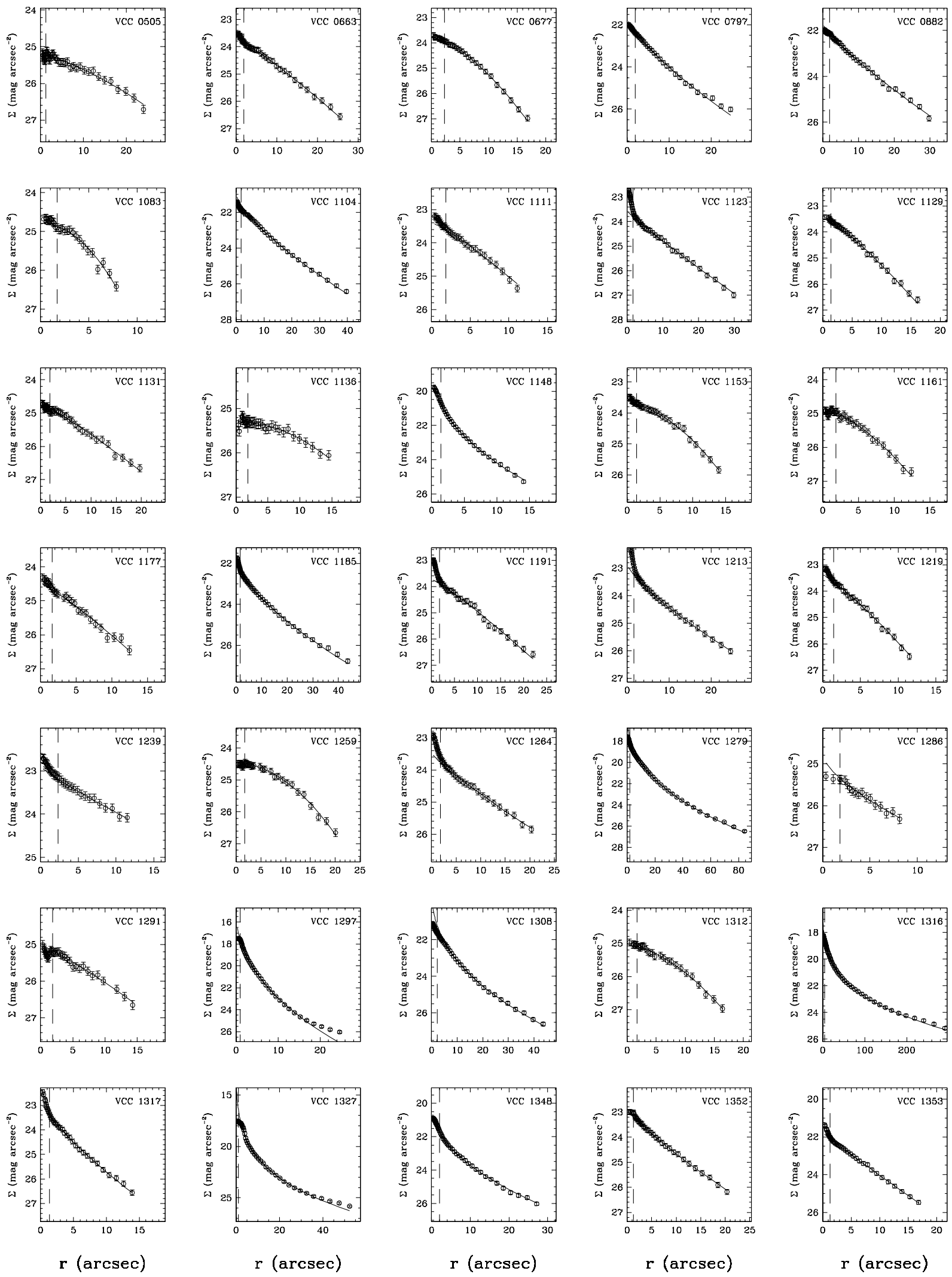

Fig. 13. The 136 individual $B$-band profiles and their Sersic fit. The vertical dashed line marks the seeing. 
G. Gavazzi et al.: INT WFS in Virgo, Online Material p 3
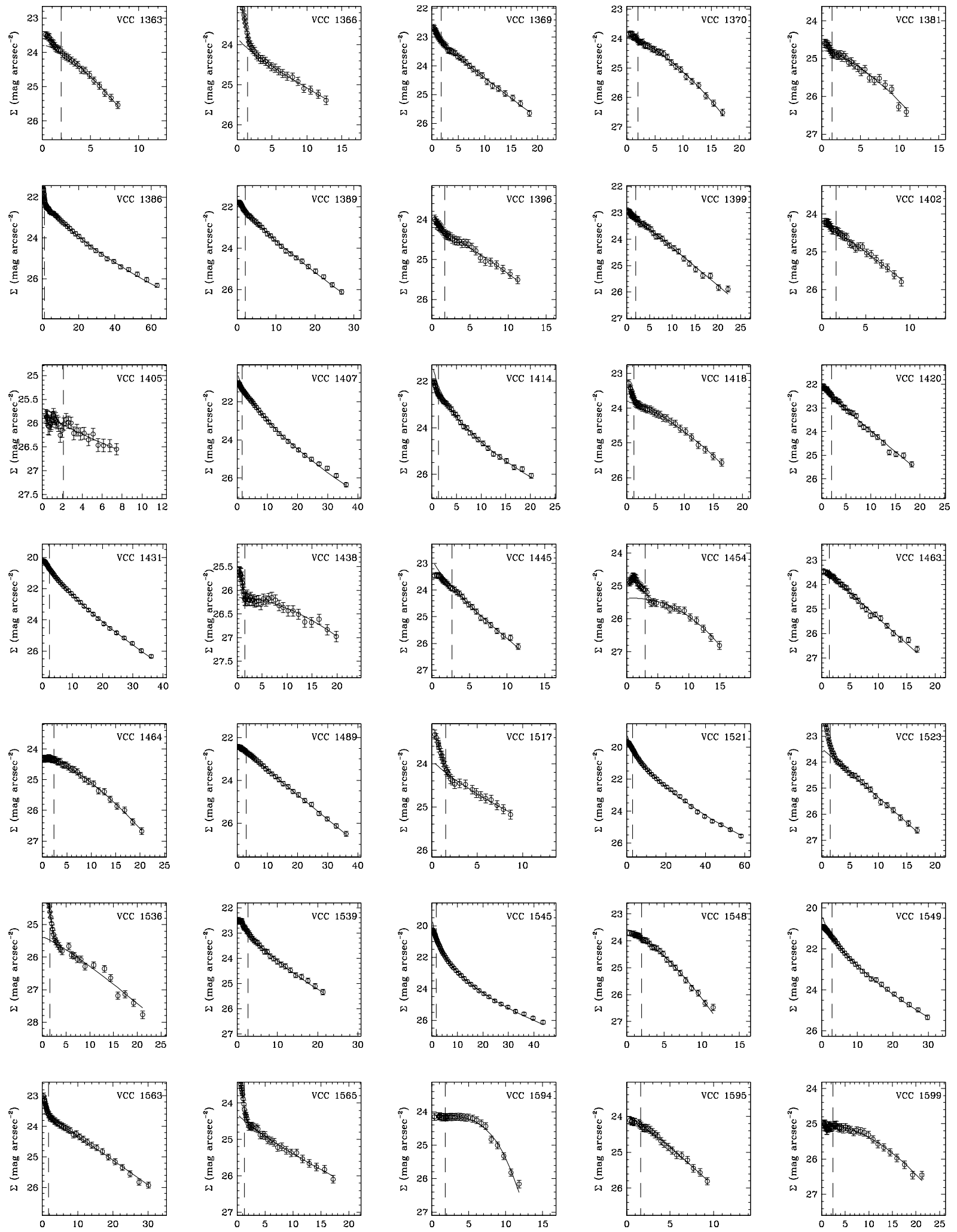

r (arcsec)

$\mathrm{r}(\operatorname{arcsec})$

r (arcsec)

r (arcsec)

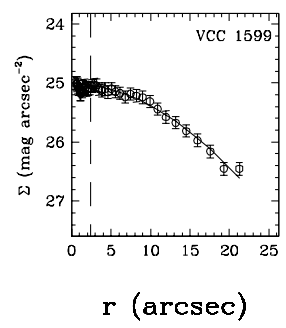

Fig. 13. continued. 
G. Gavazzi et al.: INT WFS in Virgo, Online Material p 4
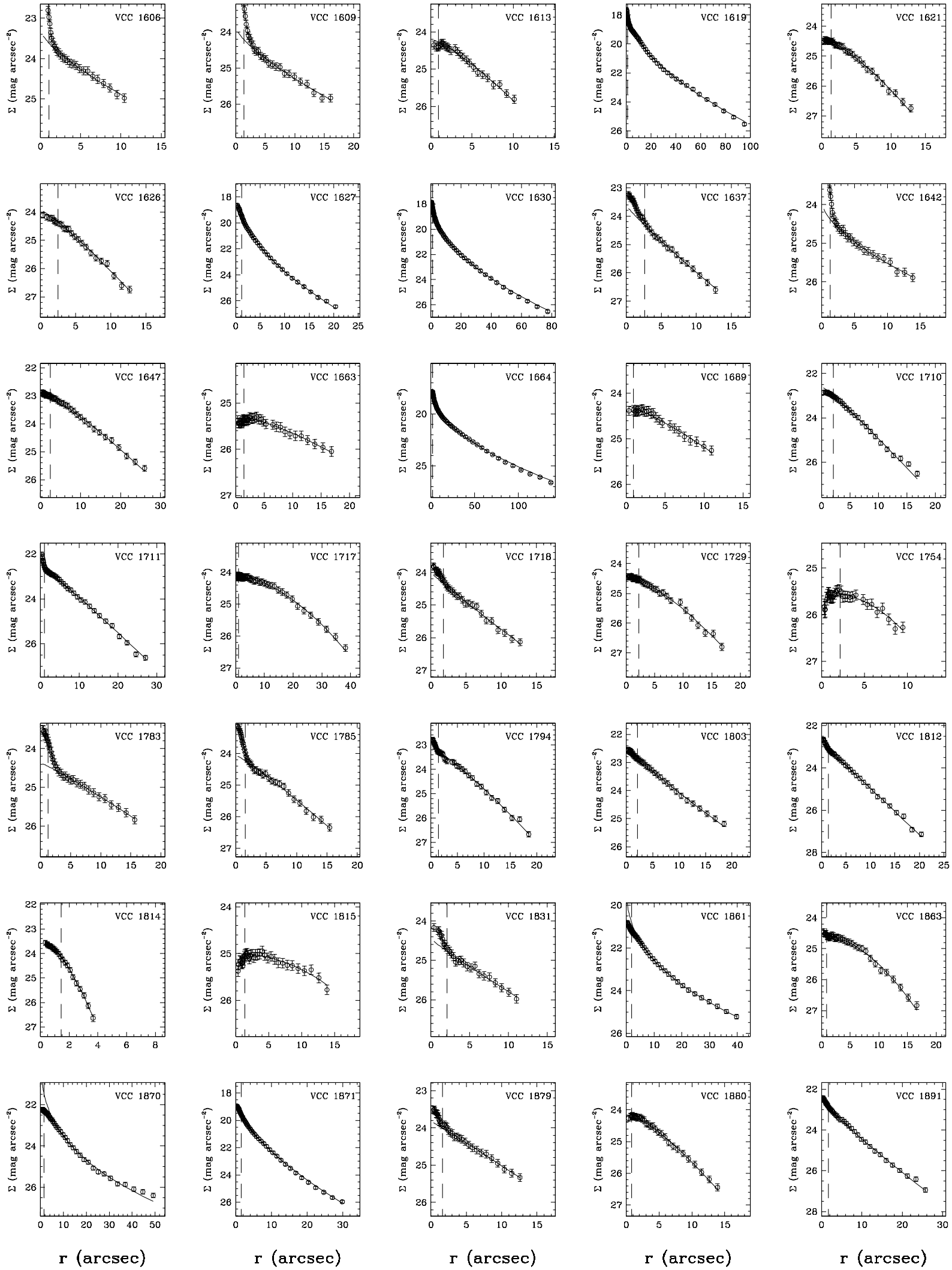

Fig. 13. continued. 
G. Gavazzi et al.: INT WFS in Virgo, Online Material p 5
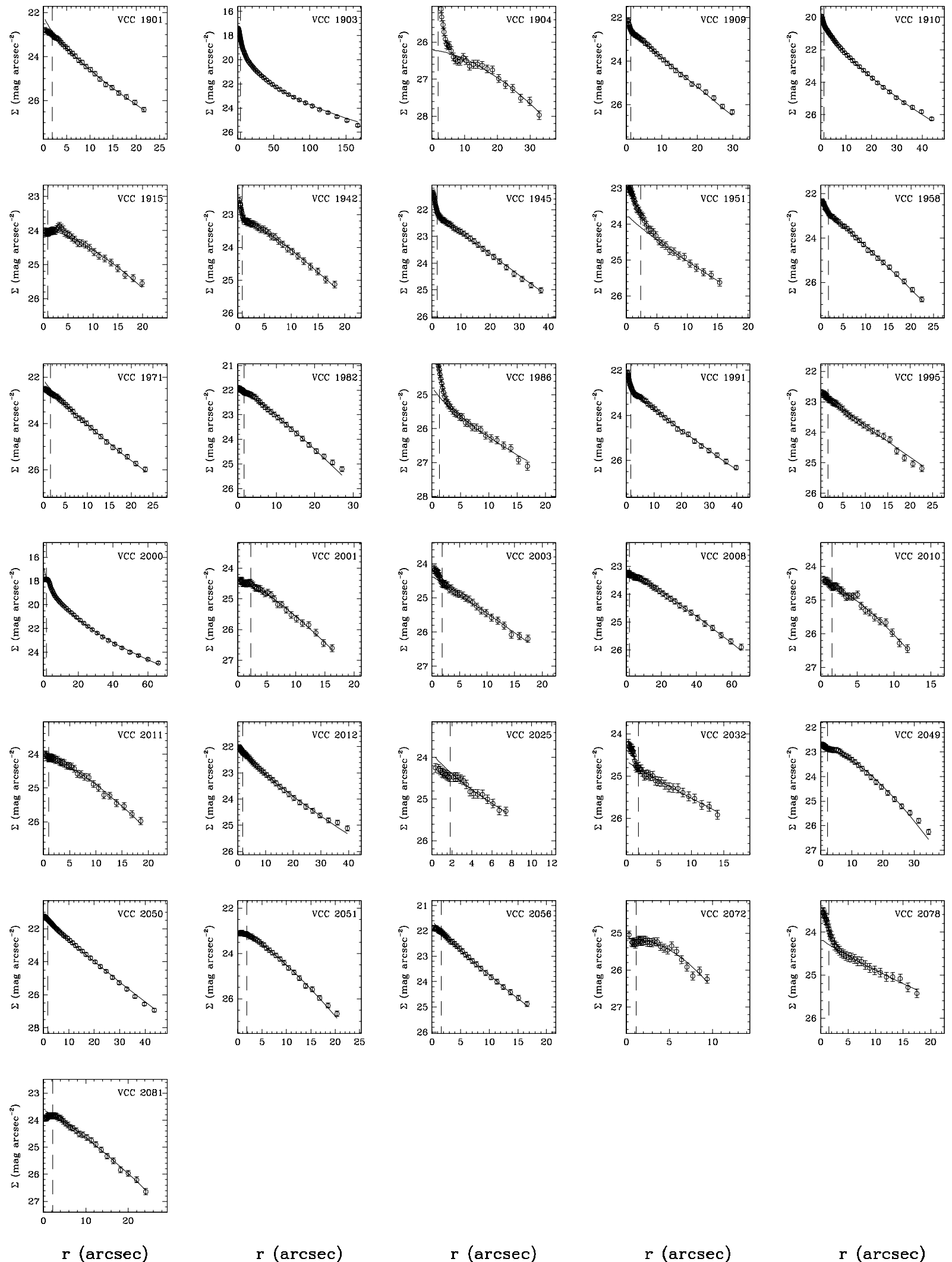

$\mathrm{r}(\operatorname{arcsec})$

$r(\operatorname{arcsec})$

$r(\operatorname{arcsec})$

$\mathrm{r}(\operatorname{arcsec})$

Fig. 13. continued. 
G. Gavazzi et al.: INT WFS in Virgo, Online Material $p 6$
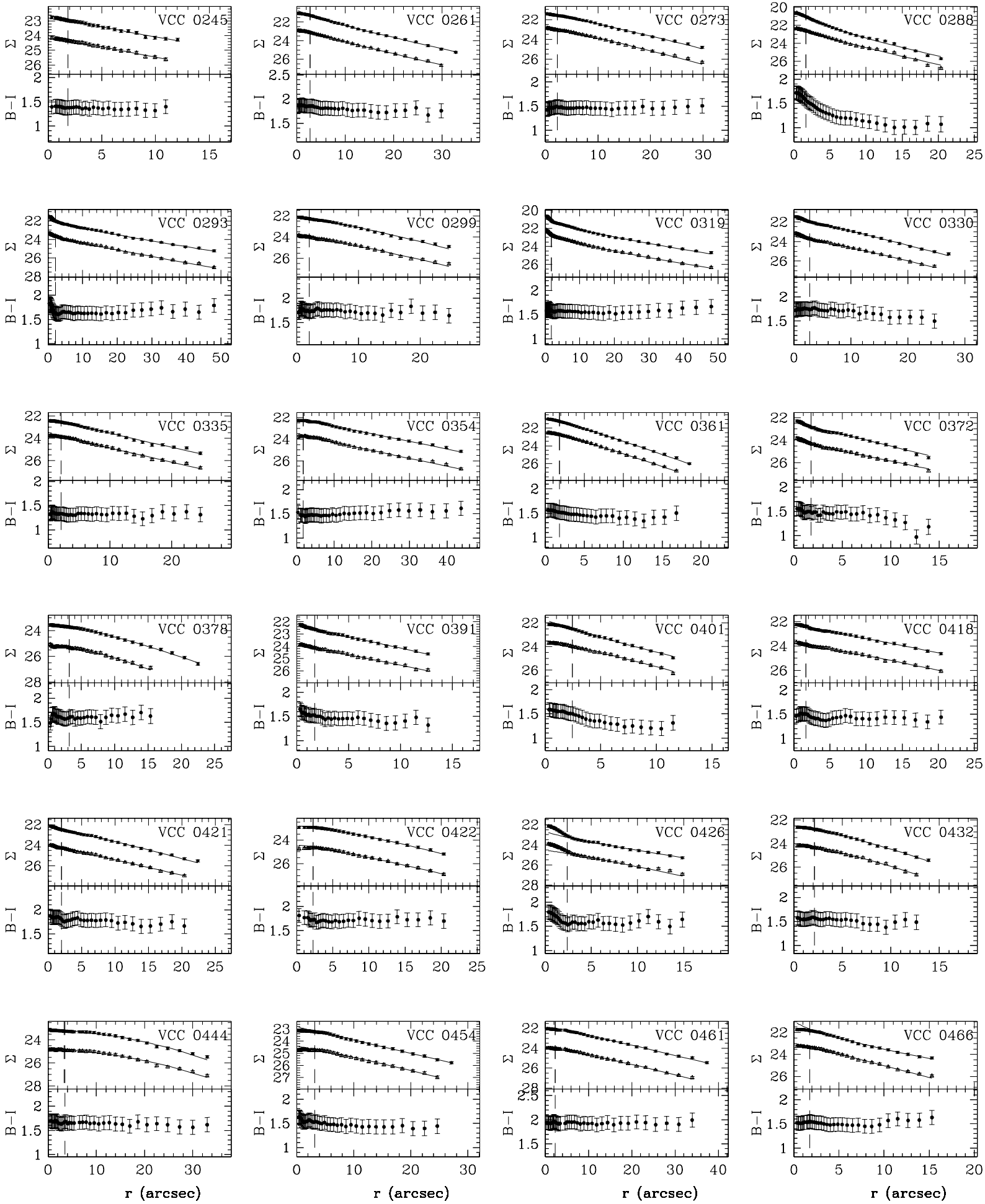

Fig. 14. The profiles and their Sersic fit for 90 galaxies in the WFV with $B$ and $I$ band imaging and their $B-I$ color profile. $I$ Images are convolved to the seeing of $B$ images, marked by the vertical dashed line. 
G. Gavazzi et al.: INT WFS in Virgo, Online Material $p 7$
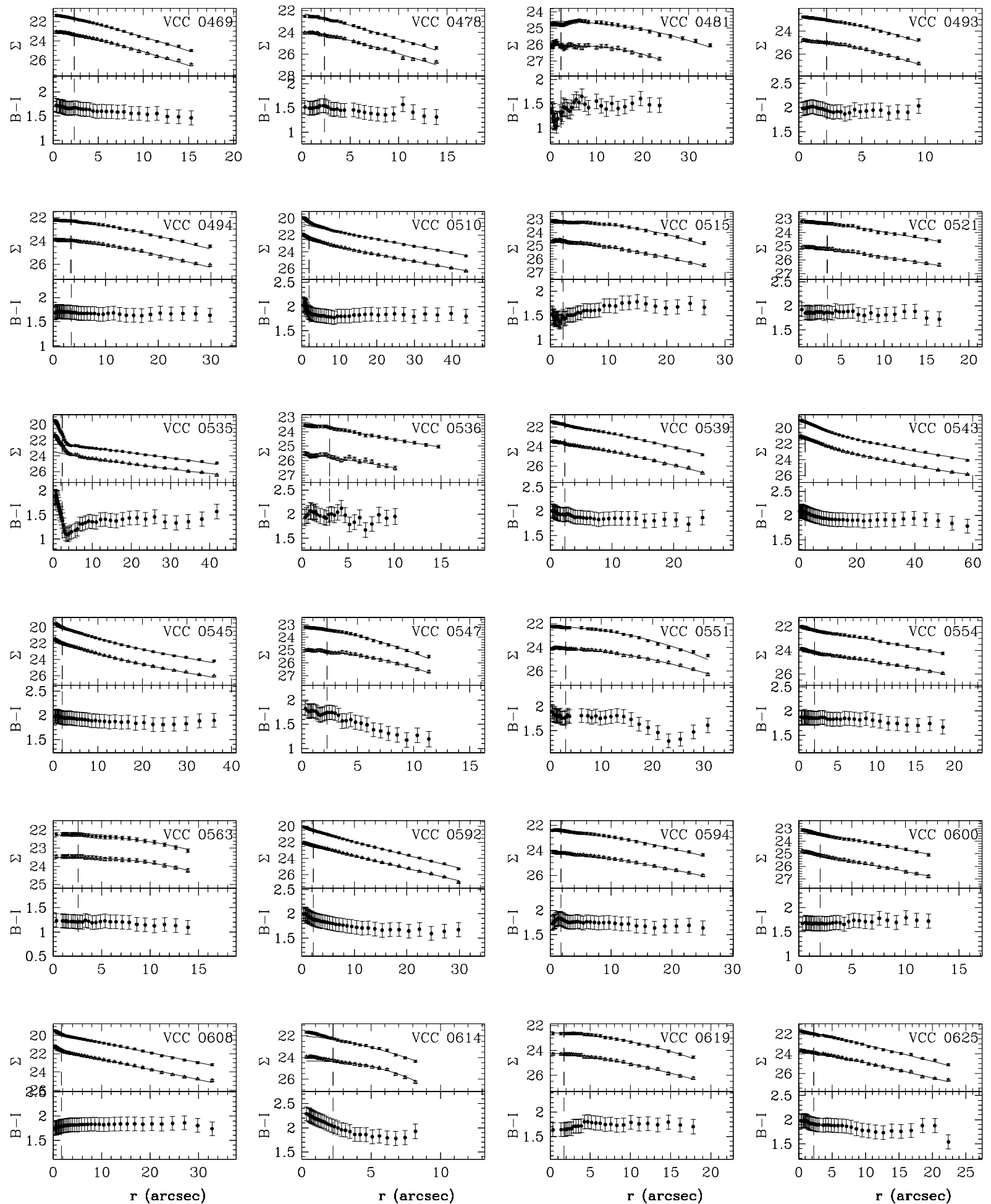

Fig. 14. continued. 
G. Gavazzi et al.: INT WFS in Virgo, Online Material $p 8$
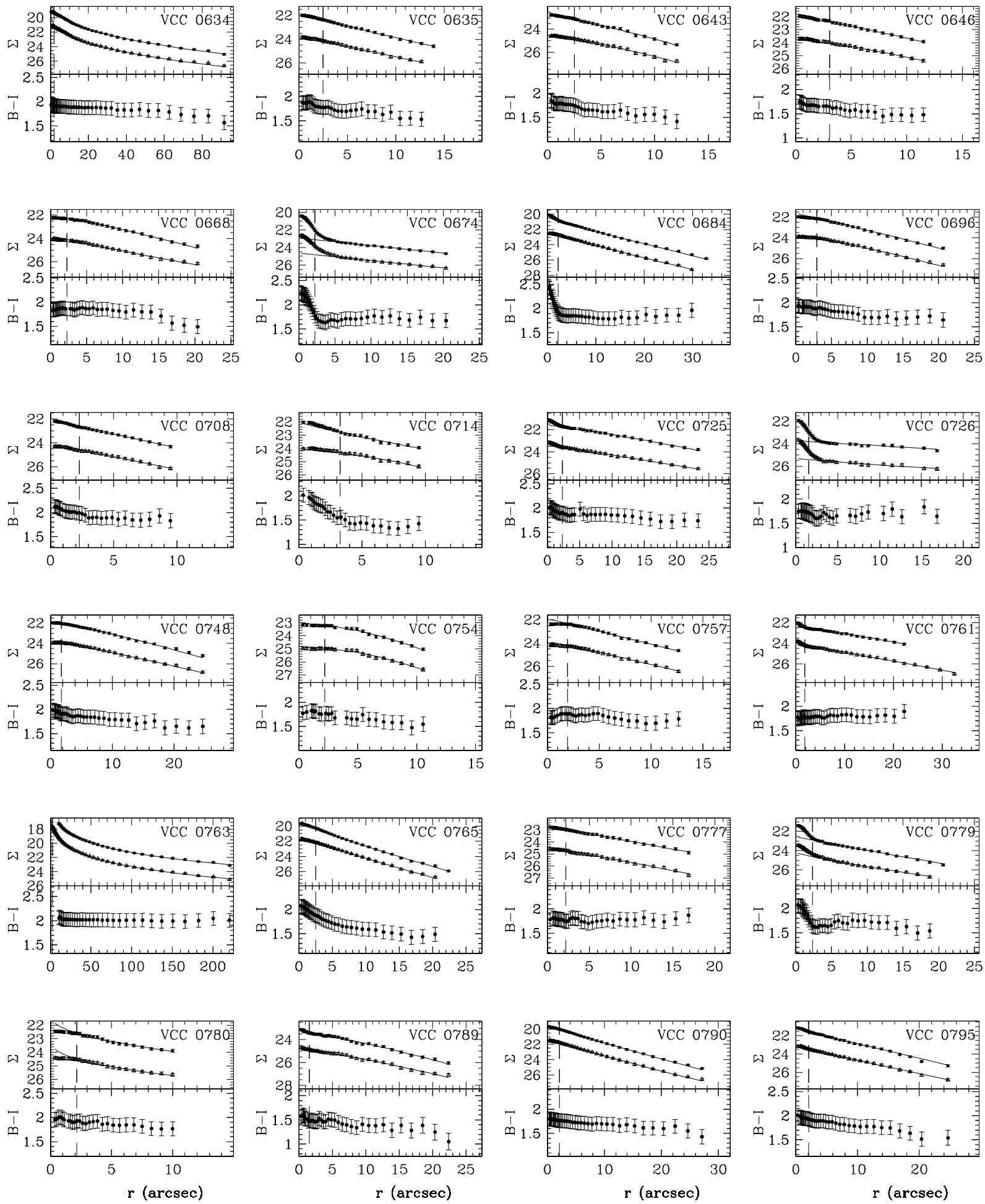

Fig. 14. continued. 
G. Gavazzi et al.: INT WFS in Virgo, Online Material p 9
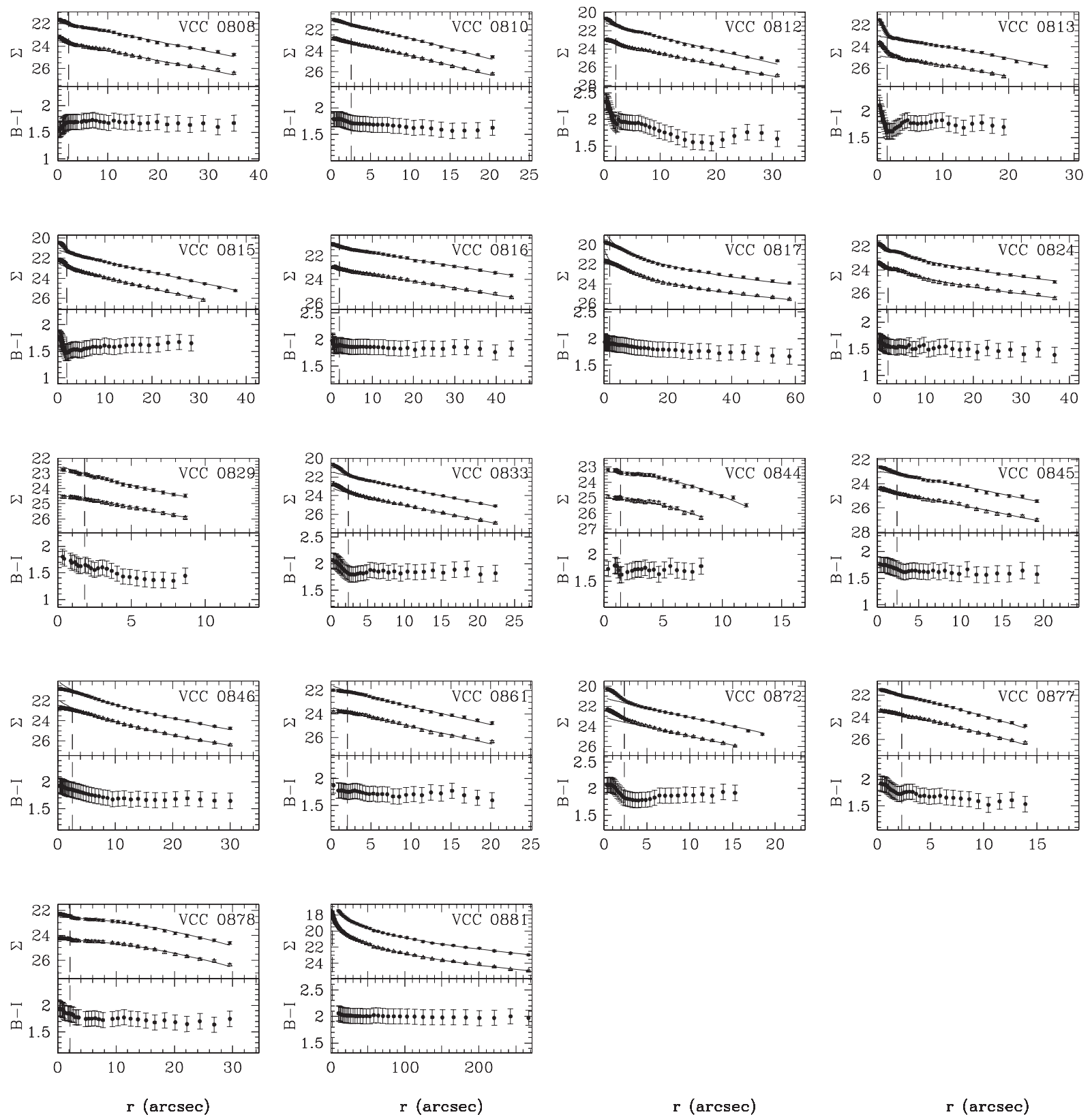

Fig. 14. continued. 
G. Gavazzi et al.: INT WFS in Virgo, Online Material p 10

Table 2: B-band parameters of the observed galaxies.

\begin{tabular}{|c|c|c|c|c|c|c|c|c|c|c|c|c|c|}
\hline $\begin{array}{l}\text { VCC } \\
\text { (1) }\end{array}$ & $\begin{array}{c}\text { Type } \\
\text { (2) }\end{array}$ & (3) & $\begin{array}{l}m_{p} \\
(4)\end{array}$ & $\begin{array}{c}n \\
(5)\end{array}$ & $\begin{array}{l}\mu_{o} \\
\text { (6) }\end{array}$ & $\begin{array}{l}B_{T} \\
(7)\end{array}$ & $\begin{array}{l}C_{31} \\
(8)\end{array}$ & $\begin{array}{c}<\mu>_{e} \\
\quad \text { (9) }\end{array}$ & $\begin{array}{r}r_{e} \\
(10)\end{array}$ & $\begin{array}{l}\text { Strip } \\
(11)\end{array}$ & $\begin{array}{c}\text { Band } \\
\text { (12) }\end{array}$ & $\begin{array}{c}\text { Notes } \\
\text { (13) }\end{array}$ & $\begin{array}{l}\text { Ref } \\
(14)\end{array}$ \\
\hline 245 & $\mathrm{dE}$ & - & 18.40 & 0.89 & 24.14 & 18.06 & 2.46 & 25.22 & 12.57 & $\mathrm{~V}$ & $\mathrm{~B}, \mathrm{I}$ & & \\
\hline 261 & $\mathrm{dE}$ & - & 16.00 & 1.11 & 22.65 & 16.56 & 3.29 & 23.89 & 13.69 & $\mathrm{~V}$ & $\mathrm{~B}, \mathrm{I}$ & & \\
\hline 273 & $\mathrm{dE}$ & $\mathrm{N}$ & 16.55 & 0.88 & 22.78 & 16.36 & 2.58 & 23.82 & 14.71 & $\mathrm{~V}$ & $\mathrm{~B}, \mathrm{I}$ & & \\
\hline 288 & $\mathrm{dE}$ & - & 17.65 & 1.22 & 22.00 & 17.33 & 3.07 & 23.37 & 8.61 & $\mathrm{~V}$ & $\mathrm{~B}, \mathrm{I}$ & 1 & \\
\hline 293 & $\mathrm{dE}$ & $\mathrm{N}$ & 16.55 & 1.10 & 23.50 & 16.35 & 2.76 & 25.06 & 28.04 & $\mathrm{~V}$ & $\mathrm{~B}, \mathrm{I}$ & & \\
\hline 299 & $\mathrm{dE}$ & - & 17.26 & 0.83 & 23.82 & 17.57 & 2.89 & 24.54 & 13.33 & $\mathrm{~V}$ & $\mathrm{~B}, \mathrm{I}$ & & BJ98 \\
\hline 319 & $\mathrm{dE}$ & $\mathrm{N}$ & 16.15 & 1.37 & 22.33 & 15.32 & 3.01 & 24.02 & 23.41 & $\mathrm{~V}$ & $\mathrm{~B}, \mathrm{I}$ & & VPC \\
\hline 330 & $\mathrm{dE}$ & $\mathrm{N}$ & 16.65 & 0.89 & 23.45 & 16.98 & 2.75 & 24.46 & 13.95 & $\mathrm{~V}$ & $\mathrm{~B}, \mathrm{I}$ & & VPC \\
\hline 335 & $\mathrm{dE}$ & - & 17.75 & 1.02 & 23.48 & 17.45 & 3.37 & 24.27 & 12.34 & V & $\mathrm{B}, \mathrm{I}$ & & \\
\hline 354 & $\mathrm{dE}$ & - & 16.55 & 1.04 & 23.44 & 16.02 & 3.37 & 24.52 & 23.65 & $\mathrm{~V}$ & $\mathrm{~B}, \mathrm{I}$ & & VPC,BJ98 \\
\hline 361 & $\mathrm{dE}$ & - & 17.35 & 0.92 & 22.27 & 17.31 & 2.75 & 23.26 & 6.57 & V & $\mathrm{B}, \mathrm{I}$ & & VPC \\
\hline 372 & $\mathrm{dE}$ & $\mathrm{N}$ & 17.95 & 1.04 & 23.95 & 18.20 & 2.76 & 25.31 & 10.82 & $\mathrm{~V}$ & $\mathrm{~B}, \mathrm{I}$ & & \\
\hline 378 & $\mathrm{dE}$ & - & 18.75 & 0.62 & 25.11 & 18.60 & 2.43 & 25.64 & 10.42 & $\mathrm{~V}$ & $\mathrm{~B}, \mathrm{I}$ & & \\
\hline 391 & $\mathrm{dE}$ & - & 17.97 & 0.95 & 23.83 & 17.88 & 2.85 & 24.96 & 10.81 & $\mathrm{~V}$ & $\mathrm{~B}, \mathrm{I}$ & & \\
\hline 401 & $\mathrm{dE}$ & - & 17.65 & 0.79 & 23.62 & 18.14 & 2.67 & 24.49 & 7.79 & $\mathrm{~V}$ & $\mathrm{~B}, \mathrm{I}$ & & \\
\hline 418 & $\mathrm{dE}$ & - & 17.84 & 0.88 & 23.75 & 17.41 & 2.84 & 24.76 & 15.53 & $\mathrm{~V}$ & $\mathrm{~B}, \mathrm{I}$ & & \\
\hline 421 & $\mathrm{dE}$ & - & 16.95 & 0.94 & 23.99 & 17.82 & 2.64 & 25.20 & 12.76 & $\mathrm{~V}$ & $\mathrm{~B}, \mathrm{I}$ & & \\
\hline 422 & $\mathrm{dE}$ & - & 17.95 & 0.83 & 24.37 & 17.88 & 2.76 & 25.42 & 14.02 & $\mathrm{~V}$ & $\mathrm{~B}, \mathrm{I}$ & & \\
\hline 426 & $\mathrm{dE}$ & $\mathrm{N}$ & 18.12 & 0.88 & 24.51 & 18.53 & 2.47 & 25.49 & 10.15 & V & $\mathrm{B}, \mathrm{I}$ & & \\
\hline 432 & $\mathrm{dE}$ & - & 18.95 & 0.75 & 24.05 & 19.04 & 3.39 & 24.95 & 7.53 & V & $\mathrm{B}, \mathrm{I}$ & & \\
\hline 444 & $\mathrm{dE}$ & - & 17.17 & 0.55 & 24.77 & 17.61 & 2.10 & 25.46 & 19.94 & V & $\mathrm{B}, \mathrm{I}$ & & BJ98 \\
\hline 454 & $\mathrm{dE}$ & - & 17.55 & 0.89 & 24.43 & 17.88 & 3.12 & 25.40 & 16.11 & V & $\mathrm{B}, \mathrm{I}$ & & \\
\hline 461 & $\mathrm{dE}$ & - & 16.45 & 0.87 & 23.79 & 16.92 & 2.68 & 24.89 & 18.74 & V & $\mathrm{B}, \mathrm{I}$ & & VPC \\
\hline 466 & $\mathrm{dE}$ & - & 18.07 & 0.95 & 22.98 & 17.64 & 2.76 & 23.97 & 8.27 & $\mathrm{~V}$ & $\mathrm{~B}, \mathrm{I}$ & & VPC \\
\hline 469 & $\mathrm{dE}$ & - & 18.50 & 0.80 & 23.00 & 17.99 & 2.67 & 23.82 & 7.25 & $\mathrm{~V}$ & $\mathrm{~B}, \mathrm{I}$ & & \\
\hline 478 & $\mathrm{dE}$ & - & 18.58 & 0.80 & 23.90 & 18.94 & 2.54 & 24.53 & 6.77 & $\mathrm{~V}$ & $\mathrm{~B}, \mathrm{I}$ & & \\
\hline 481 & $\mathrm{dE}$ & - & 18.30 & 0.34 & 26.05 & 18.54 & 1.71 & 26.45 & 19.20 & $\mathrm{~V}$ & $\mathrm{~B}, \mathrm{I}$ & & \\
\hline 493 & $\mathrm{dE}$ & - & 18.95 & 0.63 & 24.73 & 19.73 & 2.34 & 25.30 & 6.23 & $\mathrm{~V}$ & $\mathrm{~B}, \mathrm{I}$ & & \\
\hline 494 & $\mathrm{dE}$ & - & 16.59 & 0.68 & 23.82 & 16.68 & 2.33 & 24.59 & 19.03 & $\mathrm{~V}$ & $\mathrm{~B}, \mathrm{I}$ & & VPC,BJ98 \\
\hline 505 & $\mathrm{dE}$ & $\mathrm{N}$ & 17.75 & 0.72 & 25.17 & 17.61 & 2.37 & 26.07 & 24.79 & $\mathrm{~V}$ & B & & \\
\hline 510 & $\mathrm{dE}$ & $\mathrm{N}$ & 15.07 & 1.11 & 22.33 & 15.20 & 3.14 & 23.98 & 23.90 & V & $\mathrm{B}, \mathrm{I}$ & & BJ98 \\
\hline 515 & $\mathrm{dE}$ & - & 18.15 & 0.70 & 24.58 & 17.14 & 2.44 & 25.24 & 19.81 & V & $\mathrm{B}, \mathrm{I}$ & & \\
\hline 521 & $\mathrm{dE}$ & - & 18.45 & 0.97 & 24.83 & 18.22 & 3.21 & 26.00 & 19.56 & $\mathrm{~V}$ & $\mathrm{~B}, \mathrm{I}$ & & \\
\hline 535 & $\mathrm{dE}$ & - & 16.95 & 1.22 & 23.38 & 16.28 & 3.82 & 24.31 & 23.81 & $\mathrm{~V}$ & $\mathrm{~B}, \mathrm{I}$ & & \\
\hline 536 & $\mathrm{dE}$ & - & 18.95 & 0.31 & 25.72 & 19.83 & 1.91 & 25.83 & 7.58 & $\mathrm{~V}$ & $\mathrm{~B}, \mathrm{I}$ & & \\
\hline 539 & $\mathrm{dE}$ & $\mathrm{N}$ & 16.75 & 0.73 & 23.60 & 17.01 & 2.62 & 24.22 & 12.80 & $\mathrm{~V}$ & $\mathrm{~B}, \mathrm{I}$ & & VPC \\
\hline 543 & $\mathrm{dE}$ & - & 14.33 & 1.75 & 20.21 & 14.51 & 3.59 & 22.54 & 21.30 & $\mathrm{~V}$ & $\mathrm{~B}, \mathrm{I}$ & & ACS,BJ98 \\
\hline 545 & $\mathrm{dE}$ & $\mathrm{N}$ & 15.19 & 1.64 & 20.75 & 15.53 & 3.22 & 22.86 & 13.49 & $\mathrm{~V}$ & $\mathrm{~B}, \mathrm{I}$ & 3 & \\
\hline 547 & $\mathrm{dE}$ & - & 18.75 & 0.52 & 25.00 & 19.28 & 2.26 & 25.36 & 7.61 & $\mathrm{~V}$ & $\mathrm{~B}, \mathrm{I}$ & & \\
\hline 551 & $\mathrm{dE}$ & - & 16.30 & 0.55 & 24.06 & 16.78 & 2.42 & 25.16 & 21.61 & $\mathrm{~V}$ & $\mathrm{~B}, \mathrm{I}$ & & \\
\hline 554 & $\mathrm{dE}$ & $\mathrm{N}$ & 17.06 & 0.85 & 24.05 & 17.24 & 2.60 & 24.94 & 15.96 & $\mathrm{~V}$ & $\mathrm{~B}, \mathrm{I}$ & & BJ98 \\
\hline 563 & $\mathrm{dE}$ & - & 16.25 & 0.36 & 23.46 & 17.05 & 1.86 & 23.78 & 11.83 & $\mathrm{~V}$ & $\mathrm{~B}, \mathrm{I}$ & & \\
\hline 592 & $\mathrm{dE}$ & $\mathrm{N}$ & 16.55 & 1.05 & 21.95 & 16.55 & 3.29 & 22.94 & 10.64 & $\mathrm{~V}$ & $\mathrm{~B}, \mathrm{I}$ & & VPC \\
\hline 594 & $\mathrm{dE}$ & - & 17.08 & 0.68 & 24.14 & 17.40 & 2.50 & 24.81 & 18.41 & $\mathrm{~V}$ & $\mathrm{~B}, \mathrm{I}$ & & BJ98 \\
\hline 600 & $\mathrm{dE}$ & - & 17.95 & 0.91 & 24.86 & 19.05 & 2.41 & 25.89 & 11.12 & $\mathrm{~V}$ & $\mathrm{~B}, \mathrm{I}$ & & \\
\hline 608 & $\mathrm{dE}$ & $\mathrm{N}$ & 14.86 & 0.94 & 21.53 & 14.94 & 2.64 & 22.92 & 18.27 & $\mathrm{~V}$ & $\mathrm{~B}, \mathrm{I}$ & & BJ98 \\
\hline 614 & $\mathrm{dE}$ & - & 18.55 & 0.39 & 24.28 & 19.17 & 2.30 & 24.47 & 4.84 & $\mathrm{~V}$ & $\mathrm{~B}, \mathrm{I}$ & & \\
\hline 619 & $\mathrm{dE}$ & - & 18.15 & 0.65 & 24.17 & 18.09 & 2.26 & 24.81 & 11.79 & $\mathrm{~V}$ & $\mathrm{~B}, \mathrm{I}$ & & \\
\hline 625 & $\mathrm{dE}$ & - & 18.15 & 0.88 & 23.59 & 17.76 & 2.85 & 24.70 & 12.47 & $\mathrm{~V}$ & $\mathrm{~B}, \mathrm{I}$ & & \\
\hline 634 & $\mathrm{dE}$ & $\mathrm{N}$ & 14.08 & 2.78 & 18.87 & 13.99 & 4.09 & 22.92 & 27.61 & $\mathrm{~V}$ & $\mathrm{~B}, \mathrm{I}$ & & \\
\hline 635 & $\mathrm{dE}$ & - & 18.95 & 1.09 & 23.56 & 18.44 & 3.62 & 25.40 & 12.40 & $\mathrm{~V}$ & $\mathrm{~B}, \mathrm{I}$ & & \\
\hline 643 & $\mathrm{dE}$ & - & 18.45 & 0.76 & 24.50 & 19.05 & 2.87 & 25.25 & 7.57 & $\mathrm{~V}$ & $\mathrm{~B}, \mathrm{I}$ & & \\
\hline 646 & $\mathrm{dE}$ & - & 18.75 & 0.76 & 23.70 & 17.70 & 2.65 & 24.53 & 10.07 & $\mathrm{~V}$ & $\mathrm{~B}, \mathrm{I}$ & & \\
\hline 663 & $\mathrm{dE}$ & - & 18.15 & 0.86 & 23.67 & 17.28 & 2.91 & 24.48 & 14.53 & $\mathrm{~V}$ & B & & \\
\hline 668 & $\mathrm{dE}$ & - & 16.45 & 1.08 & 23.70 & 17.42 & 3.39 & 24.95 & 14.49 & $\mathrm{~V}$ & $\mathrm{~B}, \mathrm{I}$ & & VPC \\
\hline 674 & $\mathrm{dE}$ & $\mathrm{N}$ & 17.95 & 1.00 & 24.59 & 17.14 & 3.15 & 25.79 & 22.20 & $\mathrm{~V}$ & $\mathrm{~B}, \mathrm{I}$ & & \\
\hline 677 & $\mathrm{dE}$ & - & 18.15 & 0.65 & 23.74 & 17.85 & 2.32 & 24.46 & 8.56 & $\mathrm{~V}$ & B & & \\
\hline 684 & $\mathrm{dE}$ & $\mathrm{N}$ & 15.98 & 1.11 & 22.15 & 16.32 & 2.79 & 23.55 & 11.37 & V & $\mathrm{B}, \mathrm{I}$ & & VPC,BJ98 \\
\hline 696 & $\mathrm{dE}$ & - & 18.25 & 0.84 & 23.64 & 17.75 & 2.55 & 24.41 & 10.83 & $\mathrm{~V}$ & $\mathrm{~B}, \mathrm{I}$ & & \\
\hline 708 & $\mathrm{dE}$ & $\mathrm{N}$ & 18.95 & 1.00 & 24.05 & 18.81 & 2.51 & 25.04 & 7.48 & $\mathrm{~V}$ & $\mathrm{~B}, \mathrm{I}$ & & \\
\hline 714 & $\mathrm{dE}$ & - & 18.25 & 0.68 & 24.00 & 18.28 & 2.44 & 24.70 & 9.03 & $\mathrm{~V}$ & $\mathrm{~B}, \mathrm{I}$ & & \\
\hline 725 & $\mathrm{dE}$ & $\mathrm{N}$ & 15.95 & 0.94 & 23.39 & 16.20 & 2.35 & 24.74 & 21.08 & $\mathrm{~V}$ & $\mathrm{~B}, \mathrm{I}$ & & \\
\hline 726 & $\mathrm{dE}$ & $\mathrm{N}$ & 18.65 & 1.16 & 25.24 & 19.16 & 2.09 & 25.87 & 12.14 & V & $\mathrm{B}, \mathrm{I}$ & & \\
\hline 748 & $\mathrm{dE}$ & - & 17.25 & 0.89 & 23.68 & 17.53 & 3.04 & 24.73 & 13.20 & V & $\mathrm{B}, \mathrm{I}$ & & VPC \\
\hline 754 & $\mathrm{dE}$ & - & 18.95 & 0.54 & 24.80 & 19.34 & 1.95 & 25.35 & 7.49 & $\mathrm{~V}$ & $\mathrm{~B}, \mathrm{I}$ & & \\
\hline 757 & $\mathrm{dE}$ & - & 18.55 & 0.93 & 23.82 & 18.58 & 2.89 & 24.91 & 8.53 & $\mathrm{~V}$ & $\mathrm{~B}, \mathrm{I}$ & & \\
\hline 761 & $\mathrm{dE}$ & - & 17.21 & 0.95 & 24.06 & 17.22 & 3.06 & 25.11 & 21.51 & $\mathrm{~V}$ & $\mathrm{~B}, \mathrm{I}$ & & BJ98 \\
\hline 763 M84 & $\mathrm{E}$ & - & 10.20 & 7.14 & 8.74 & 10.22 & 5.29 & 20.61 & 48.55 & V & $\mathrm{B}, \mathrm{I}$ & $3,6,4$ & ACS* \\
\hline 765 & $\mathrm{dE}$ & $\mathrm{N}$ & 16.43 & 1.12 & 21.19 & 16.49 & 2.79 & 22.54 & 6.72 & $\mathrm{~V}$ & $\mathrm{~B}, \mathrm{I}$ & & VPC,BJ98 \\
\hline 777 & $\mathrm{dE}$ & - & 17.95 & 0.88 & 24.50 & 17.91 & 2.65 & 25.53 & 14.17 & $\mathrm{~V}$ & $\mathrm{~B}, \mathrm{I}$ & & \\
\hline 779 & $\mathrm{dE}$ & $\mathrm{N}$ & 17.62 & 1.25 & 24.10 & 17.74 & 3.56 & 25.54 & 15.42 & $\mathrm{~V}$ & $\mathrm{~B}, \mathrm{I}$ & & BJ98 \\
\hline 780 & $\mathrm{dE}$ & - & 18.27 & 2.50 & 22.99 & 18.89 & 5.75 & 25.11 & 7.53 & $\mathrm{~V}$ & $\mathrm{~B}, \mathrm{I}$ & & \\
\hline 789 & $\mathrm{dE}$ & - & 18.95 & 0.81 & 24.76 & 18.54 & 2.92 & 25.71 & 14.69 & V & $\mathrm{B}, \mathrm{I}$ & & \\
\hline 790 & $\mathrm{dE}$ & $\mathrm{N}$ & 16.34 & 1.14 & 21.07 & 15.90 & 2.88 & 22.70 & 9.53 & V & $\mathrm{B}, \mathrm{I}$ & & VPC \\
\hline 795 & $\mathrm{dE}$ & $\mathrm{N}$ & 17.95 & 1.05 & 23.08 & 17.60 & 3.19 & 24.09 & 11.17 & V & $\mathrm{B}, \mathrm{I}$ & & VPC \\
\hline 797 & $\mathrm{dE}$ & $\mathrm{N}$ & 16.94 & 1.33 & 21.59 & 16.49 & 3.26 & 22.92 & 9.32 & $\mathrm{~V}$ & B & & \\
\hline 808 & $\mathrm{dE}$ & $\mathrm{N}$ & 17.65 & 0.99 & 23.54 & 16.74 & 3.10 & 24.46 & 19.25 & $\mathrm{~V}$ & $\mathrm{~B}, \mathrm{I}$ & & VPC \\
\hline 810 & $\mathrm{dE}$ & $\mathrm{N}$ & 16.91 & 0.91 & 22.86 & 16.86 & 2.68 & 23.71 & 9.67 & $\mathrm{~V}$ & $\mathrm{~B}, \mathrm{I}$ & & B03,VPC,BJ98 \\
\hline 812 & $\mathrm{dE}$ & $\mathrm{N}$ & 16.98 & 1.10 & 23.00 & 16.88 & 2.89 & 24.16 & 13.15 & $\mathrm{~V}$ & $\mathrm{~B}, \mathrm{I}$ & & VPC \\
\hline 813 & $\mathrm{dE}$ & $\mathrm{N}$ & 18.15 & 0.93 & 24.78 & 17.59 & 2.84 & 25.75 & 18.01 & $\mathrm{~V}$ & $\mathrm{~B}, \mathrm{I}$ & & \\
\hline 815 & $\mathrm{dE}$ & $\mathrm{N}$ & 16.06 & 1.45 & 22.13 & 16.16 & 3.12 & 23.87 & 15.61 & $\mathrm{~V}$ & $\mathrm{~B}, \mathrm{I}$ & & B03.VPC,BJ98 \\
\hline
\end{tabular}


Table 2: Continued

\begin{tabular}{|c|c|c|c|c|c|c|c|c|c|c|c|c|c|}
\hline $\begin{array}{l}\text { VCC } \\
\text { (1) }\end{array}$ & $\begin{array}{l}\text { Type } \\
\text { (2) }\end{array}$ & (3) & $\begin{array}{l}m_{p} \\
(4)\end{array}$ & $\begin{array}{c}n \\
(5)\end{array}$ & $\begin{array}{l}\mu_{o} \\
\text { (6) }\end{array}$ & $\begin{array}{l}B_{T} \\
\text { (7) }\end{array}$ & $\begin{array}{l}C_{31} \\
(8)\end{array}$ & $\begin{array}{c}<\mu>_{e} \\
\quad \text { (9) }\end{array}$ & $\begin{array}{r}r_{e} \\
(10)\end{array}$ & $\begin{array}{c}\text { Strip } \\
(11)\end{array}$ & $\begin{array}{c}\text { Band } \\
(12)\end{array}$ & $\begin{array}{c}\text { Notes } \\
\text { (13) }\end{array}$ & $\begin{array}{l}\text { Ref } \\
(14)\end{array}$ \\
\hline 816 & $\mathrm{dE}$ & $\mathrm{N}$ & 15.25 & 0.97 & 23.01 & 15.18 & 2.53 & 24.29 & 33.51 & $\mathrm{~V}$ & $\mathrm{~B}, \mathrm{I}$ & & \\
\hline 817 & $\mathrm{dE}$ & - & 14.93 & 3.70 & 18.92 & 14.66 & 3.35 & 23.05 & 21.35 & V & $\mathrm{B}, \mathrm{I}$ & & \\
\hline 824 & $\mathrm{dE}$ & $\mathrm{N}$ & 17.95 & 2.08 & 22.77 & 16.56 & 3.09 & 25.13 & 23.64 & $\mathrm{~V}$ & $\mathrm{~B}, \mathrm{I}$ & & \\
\hline 829 & $\mathrm{dE}$ & - & 18.45 & 0.90 & 24.39 & 19.01 & 2.89 & 25.34 & 9.59 & $\mathrm{~V}$ & $\mathrm{~B}, \mathrm{I}$ & & \\
\hline 833 & $\mathrm{dE}$ & $\mathrm{N}$ & 17.42 & 1.15 & 23.12 & 17.24 & 3.04 & 24.34 & 10.97 & $\mathrm{~V}$ & $\mathrm{~B}, \mathrm{I}$ & & VPC \\
\hline 844 & $\mathrm{dE}$ & - & 18.85 & 0.52 & 24.98 & 19.77 & 2.20 & 25.50 & 6.61 & $\mathrm{~V}$ & $\mathrm{~B}, \mathrm{I}$ & & \\
\hline 845 & $\mathrm{dE}$ & - & 18.95 & 0.92 & 24.52 & 18.77 & 2.89 & 25.28 & 11.71 & $\mathrm{~V}$ & $\mathrm{~B}, \mathrm{I}$ & & \\
\hline 846 & $\mathrm{dE}$ & $\mathrm{N}$ & 16.16 & 1.61 & 21.64 & 16.39 & 3.11 & 23.61 & 12.50 & $\mathrm{~V}$ & $\mathrm{~B}, \mathrm{I}$ & & B03,VPC \\
\hline 861 & $\mathrm{dE}$ & - & 17.85 & 1.06 & 23.38 & 17.72 & 3.15 & 24.34 & 10.90 & V & $\mathrm{B}, \mathrm{I}$ & & \\
\hline 872 & $\mathrm{dE}$ & $\mathrm{N}$ & 16.93 & 1.04 & 23.04 & 17.23 & 3.25 & 23.92 & 9.21 & V & $\mathrm{B}, \mathrm{I}$ & & BJ98 \\
\hline 877 & $\mathrm{dE}$ & $\mathrm{N}$ & 17.55 & 0.83 & 23.39 & 17.90 & 2.58 & 24.22 & 7.56 & $\mathrm{~V}$ & $\mathrm{~B}, \mathrm{I}$ & & \\
\hline 878 & $\mathrm{dE}$ & - & 17.25 & 0.57 & 24.30 & 17.15 & 2.13 & 24.83 & 18.44 & $\mathrm{~V}$ & $\mathrm{~B}, \mathrm{I}$ & & \\
\hline 881 M86 & $\mathrm{E}$ & - & 10.01 & 3.45 & 16.31 & 10.19 & 5.15 & 20.89 & 64.16 & $\mathrm{~V}$ & $\mathrm{~B}, \mathrm{I}$ & 3,4 & ACS* \\
\hline 882 & $\mathrm{dE}$ & $\mathrm{N}$ & 16.63 & 1.19 & 21.57 & 15.76 & 2.99 & 22.99 & 12.88 & V & B & & \\
\hline 1083 & $\mathrm{dE}$ & - & 19.00 & 0.49 & 24.75 & 19.89 & 2.13 & 25.15 & 5.38 & $\mathrm{H}$ & B & & \\
\hline 1104 & $\mathrm{dE}$ & $\mathrm{N}$ & 15.14 & 1.08 & 21.45 & 15.42 & 3.12 & 22.61 & 13.30 & $\mathrm{H}$ & B & 3 & B03,VPC,BJ98 \\
\hline 1111 & $\mathrm{dE}$ & $\mathrm{N}$ & 17.65 & 0.84 & 23.34 & 17.62 & 2.45 & 24.34 & 9.85 & $\mathrm{H}$ & B & & \\
\hline 1123 & $\mathrm{dE}$ & $\mathrm{N}$ & 16.60 & 1.09 & 23.51 & 16.79 & 2.83 & 24.95 & 18.22 & $\mathrm{H}$ & B & & VPC \\
\hline 1129 & $\mathrm{dE}$ & - & 17.64 & 0.81 & 23.36 & 17.82 & 2.50 & 24.28 & 8.36 & $\mathrm{H}$ & B & & B03 \\
\hline 1131 & $\mathrm{dE}$ & - & 18.05 & 0.87 & 24.67 & 18.01 & 3.07 & 25.45 & 14.89 & $\mathrm{H}$ & B & & \\
\hline 1136 & $\mathrm{dE}$ & $\mathrm{N}$ & 17.97 & 0.49 & 25.25 & 18.53 & 2.09 & 25.84 & 14.65 & $\mathrm{H}$ & B & & \\
\hline 1148 & $\mathrm{E}$ & - & 15.90 & 2.22 & 18.32 & 16.21 & 3.60 & 21.21 & 4.11 & $\mathrm{H}$ & B & & VPC \\
\hline 1153 & $\mathrm{dE}$ & - & 17.75 & 0.57 & 23.64 & 17.97 & 2.19 & 24.29 & 9.22 & $\mathrm{H}$ & B & & \\
\hline 1161 & $\mathrm{dE}$ & - & 18.98 & 0.65 & 24.83 & 19.18 & 2.32 & 25.45 & 8.62 & $\mathrm{H}$ & B & & \\
\hline 1177 & $\mathrm{dE}$ & - & 18.65 & 0.85 & 24.44 & 18.67 & 2.77 & 25.60 & 10.87 & $\mathrm{H}$ & B & & \\
\hline 1185 & $\mathrm{dE}$ & $\mathrm{N}$ & 15.62 & 1.52 & 21.72 & 15.57 & 3.17 & 23.55 & 16.76 & $\mathrm{H}$ & B & & VPC,ACS,BJ98 \\
\hline 1191 & $\mathrm{dE}$ & $\mathrm{N}$ & 17.37 & 0.87 & 23.68 & 17.61 & 2.86 & 24.34 & 11.25 & $\mathrm{H}$ & B & & \\
\hline 1213 & $\mathrm{dE}$ & $\mathrm{N}$ & 16.37 & 1.32 & 22.80 & 16.37 & 3.06 & 24.24 & 15.61 & $\mathrm{H}$ & B & & VPC,BJ98 \\
\hline 1219 & $\mathrm{dE}$ & $\mathrm{N}$ & 18.16 & 0.84 & 23.34 & 18.37 & 2.88 & 24.32 & 6.71 & $\mathrm{H}$ & B & & \\
\hline 1239 & $\mathrm{dE}$ & $\mathrm{N}$ & 17.75 & 1.14 & 22.85 & 17.26 & 19.21 & 24.65 & 19.00 & $\mathrm{H}$ & B & & \\
\hline 1259 & $\mathrm{dE}$ & - & 18.19 & 0.51 & 24.43 & 18.21 & 2.16 & 24.96 & 12.36 & $\mathrm{H}$ & B & & \\
\hline 1264 & $\mathrm{dE}$ & $\mathrm{N}$ & 17.26 & 1.16 & 23.42 & 16.60 & 2.96 & 24.79 & 17.60 & $\mathrm{H}$ & B & & BJ98 \\
\hline 1279 & $\mathrm{E}$ & - & 12.08 & 2.56 & 14.96 & 12.20 & 4.13 & 19.70 & 13.68 & $\mathrm{H}$ & B & 3,5 & F97,ACS \\
\hline 1286 & $\mathrm{dE}$ & - & 18.80 & 1.49 & 24.75 & 18.93 & 4.33 & 27.08 & 19.05 & $\mathrm{H}$ & B & & \\
\hline 1291 & $\mathrm{dE}$ & - & 18.77 & 0.86 & 24.97 & 18.50 & 2.63 & 25.88 & 14.60 & $\mathrm{H}$ & B & & \\
\hline 1297 & E & - & 14.26 & 2.38 & 14.26 & 14.20 & 3.63 & 18.45 & 3.01 & $\mathrm{H}$ & B & $1,2,4$ & F97,ACS \\
\hline 1308 & $\mathrm{dE}$ & $\mathrm{N}$ & 15.56 & 2.38 & 18.98 & 15.64 & 3.49 & 22.01 & 10.69 & $\mathrm{H}$ & B & 3 & VPC,BJ98 \\
\hline 1312 & $\mathrm{dE}$ & - & 18.65 & 0.59 & 25.01 & 19.14 & 2.37 & 25.60 & 11.29 & $\mathrm{H}$ & B & & \\
\hline 1316 M87 & $\mathrm{E}$ & - & 9.51 & 3.85 & 15.07 & 9.80 & 4.95 & 20.48 & 63.53 & $\mathrm{H}$ & B & 4 & F97.ACS \\
\hline 1317 & $\mathrm{dE}$ & $\mathrm{N}$ & 17.93 & 1.28 & 22.76 & 18.13 & 3.12 & 24.03 & 6.79 & $\mathrm{H}$ & B & & \\
\hline 1327 & $\mathrm{E}$ & - & 13.20 & 5.00 & 8.10 & 13.11 & 5.12 & 19.12 & 7.01 & $\mathrm{H}$ & B & $1,2,4$ & ACS* \\
\hline 1348 & $\mathrm{dE}$ & - & 15.79 & 2.17 & 19.82 & 15.87 & 3.34 & 22.40 & 8.80 & $\mathrm{H}$ & B & & VPC,BJ98 \\
\hline 1352 & $\mathrm{dE}$ & - & 17.15 & 1.27 & 22.70 & 17.19 & 3.14 & 24.02 & 11.51 & $\mathrm{H}$ & B & & VPC \\
\hline 1353 & $\mathrm{dE}$ & $\mathrm{N}$ & 16.56 & 1.05 & 21.71 & 16.54 & 3.08 & 22.87 & 8.20 & $\mathrm{H}$ & B & & VPC,BJ98 \\
\hline 1363 & $\mathrm{dE}$ & $\mathrm{N}$ & 18.93 & 0.69 & 23.73 & 18.86 & 2.72 & 24.31 & 5.86 & $\mathrm{H}$ & B & & \\
\hline 1366 & $\mathrm{dE}$ & $\mathrm{N}$ & 17.55 & 1.23 & 23.77 & 16.98 & 3.41 & 25.25 & 19.19 & $\mathrm{H}$ & B & & \\
\hline 1369 & $\mathrm{dE}$ & $\mathrm{N}$ & 17.23 & 1.10 & 22.76 & 17.03 & 3.00 & 24.03 & 13.07 & $\mathrm{H}$ & B & & VPC \\
\hline 1370 & $\mathrm{dE}$ & - & 17.35 & 0.65 & 23.95 & 17.66 & 2.14 & 24.57 & 9.89 & $\mathrm{H}$ & B & & \\
\hline 1381 & $\mathrm{dE}$ & - & 18.95 & 0.65 & 24.74 & 18.90 & 2.27 & 25.37 & 9.12 & $\mathrm{H}$ & B & & \\
\hline 1386 & $\mathrm{dE}$ & $\mathrm{N}$ & 14.25 & 1.59 & 21.53 & 14.88 & 3.54 & 23.52 & 27.11 & $\mathrm{H}$ & B & & VPC \\
\hline 1389 & $\mathrm{dE}$ & $\mathrm{N}$ & 15.85 & 1.19 & 21.65 & 16.09 & 3.25 & 22.92 & 11.09 & $\mathrm{H}$ & B & & VPC,BJ98 \\
\hline 1396 & $\mathrm{dE}$ & $\mathrm{N}$ & 17.16 & 0.83 & 24.17 & 17.57 & 2.57 & 25.15 & 13.17 & $\mathrm{H}$ & B & & BJ98 \\
\hline 1399 & $\mathrm{dE}$ & $\mathrm{N}$ & 16.44 & 0.96 & 22.92 & 17.19 & 3.13 & 23.76 & 11.73 & $\mathrm{H}$ & B & & BJ98 \\
\hline 1402 & $\mathrm{dE}$ & $\mathrm{N}$ & 17.95 & 0.89 & 24.19 & 18.36 & 2.65 & 25.18 & 9.95 & $\mathrm{H}$ & B & & \\
\hline 1405 & $\mathrm{dE}$ & - & 18.95 & 1.27 & 25.70 & 20.76 & 1.92 & 26.15 & 5.34 & $\mathrm{H}$ & B & & \\
\hline 1407 & $\mathrm{dE}$ & $\mathrm{N}$ & 15.43 & 1.54 & 20.29 & 15.18 & 3.39 & 22.37 & 11.81 & $\mathrm{H}$ & B & 3 & VPC,ACS,BJ98 \\
\hline 1414 & $\mathrm{dE}$ & - & 16.95 & 2.17 & 20.60 & 17.42 & 3.35 & 23.15 & 7.53 & $\mathrm{H}$ & B & 3 & \\
\hline 1418 & $\mathrm{dE}$ & - & 17.37 & 0.68 & 23.77 & 17.49 & 2.37 & 24.57 & 13.03 & $\mathrm{H}$ & B & & \\
\hline 1420 & $\mathrm{dE}$ & $\mathrm{N}$ & 16.34 & 1.09 & 21.96 & 16.53 & 3.13 & 23.00 & 9.48 & $\mathrm{H}$ & B & & VPC,BJ98 \\
\hline 1431 & $\mathrm{dE}$ & $\mathrm{N}$ & 14.43 & 1.37 & 19.76 & 14.53 & 3.17 & 21.54 & 10.10 & $\mathrm{H}$ & $\mathrm{B}$ & & ACS \\
\hline 1438 & $\mathrm{dE}$ & - & 17.82 & 0.54 & 26.10 & 18.05 & 2.22 & 26.56 & 20.60 & $\mathrm{H}$ & $\mathrm{B}$ & & \\
\hline 1445 & $\mathrm{dE}$ & - & 18.37 & 1.28 & 22.76 & 18.33 & 2.88 & 24.17 & 6.56 & $\mathrm{H}$ & B & & \\
\hline 1454 & $\mathrm{dE}$ & $\mathrm{N}$ & 18.64 & 0.43 & 25.32 & 18.57 & 2.10 & 25.69 & 10.88 & $\mathrm{H}$ & B & & \\
\hline 1463 & $\mathrm{dE}$ & - & 18.45 & 1.01 & 23.22 & 18.51 & 2.93 & 23.88 & 7.29 & $\mathrm{H}$ & B & & \\
\hline 1464 & $\mathrm{dE}$ & - & 17.67 & 0.70 & 24.18 & 17.81 & 2.53 & 24.88 & 12.84 & $\mathrm{H}$ & B & & \\
\hline 1489 & $\mathrm{dE}$ & - & 15.84 & 1.03 & 22.22 & 16.04 & 3.07 & 23.24 & 14.72 & $\mathrm{H}$ & B & & VPC,ACS \\
\hline 1517 & $\mathrm{dE}$ & $\mathrm{N}$ & 17.25 & 1.30 & 23.84 & 18.01 & 2.41 & 24.86 & 10.46 & $\mathrm{H}$ & B & & \\
\hline 1521 & $\mathrm{E}$ & - & 14.14 & 1.72 & 18.87 & 14.09 & 4.19 & 20.74 & 14.51 & $\mathrm{H}$ & B & & \\
\hline 1523 & $\mathrm{dE}$ & $\mathrm{N}$ & 17.59 & 0.97 & 23.43 & 17.68 & 2.94 & 24.35 & 8.98 & $\mathrm{H}$ & B & & BJ98 \\
\hline 1536 & $\mathrm{dE}$ & $\mathrm{N}$ & 18.35 & 0.85 & 25.32 & 18.69 & 3.38 & 26.20 & 15.28 & $\mathrm{H}$ & B & & \\
\hline 1539 & $\mathrm{dE}$ & $\mathrm{N}$ & 15.61 & 1.37 & 22.31 & 16.09 & 3.03 & 23.94 & 15.67 & $\mathrm{H}$ & B & & VPC,ACS,BJ98 \\
\hline 1545 & $\mathrm{E}$ & - & 14.88 & 2.63 & 18.48 & 15.08 & 4.27 & 21.90 & 11.34 & $\mathrm{H}$ & B & & ACS \\
\hline 1548 & $\mathrm{dE}$ & - & 18.54 & 0.68 & 23.63 & 19.02 & 2.34 & 24.17 & 5.75 & $\mathrm{H}$ & B & & \\
\hline 1549 & $\mathrm{dE}$ & $\mathrm{N}$ & 14.56 & 1.67 & 20.03 & 15.07 & 3.41 & 21.93 & 10.51 & $\mathrm{H}$ & B & & VPC \\
\hline 1563 & $\mathrm{dE}$ & $\mathrm{N}$ & 16.06 & 0.97 & 23.50 & 16.27 & 2.90 & 24.54 & 22.19 & $\mathrm{H}$ & B & & VPC,BJ98 \\
\hline 1565 & $\mathrm{dE}$ & $\mathrm{N}$ & 16.86 & 1.23 & 24.22 & 17.11 & 3.36 & 25.74 & 22.57 & $\mathrm{H}$ & B & & VPC \\
\hline 1594 & $\mathrm{dE}$ & - & 18.68 & 0.28 & 24.06 & 18.91 & 1.54 & 24.59 & 7.78 & $\mathrm{H}$ & B & & \\
\hline 1595 & $\mathrm{dE}$ & - & 18.33 & 0.85 & 23.96 & 18.49 & 2.68 & 24.87 & 8.32 & $\mathrm{H}$ & B & & \\
\hline 1599 & $\mathrm{dE}$ & - & 17.25 & 0.52 & 24.96 & 17.95 & 1.96 & 25.49 & 15.50 & $\mathrm{H}$ & B & & \\
\hline 1606 & $\mathrm{dE}$ & $\mathrm{N}$ & 17.45 & 1.39 & 23.25 & 17.17 & 4.22 & 24.99 & 16.81 & $\mathrm{H}$ & B & & \\
\hline 1609 & $\mathrm{dE}$ & $\mathrm{N}$ & 17.32 & 1.37 & 23.83 & 17.14 & 3.92 & 25.34 & 18.09 & $\mathrm{H}$ & B & & \\
\hline 1613 & $\mathrm{dE}$ & - & 18.45 & 0.78 & 24.15 & 18.67 & 2.63 & 24.98 & 9.07 & $\mathrm{H}$ & B & & \\
\hline
\end{tabular}


G. Gavazzi et al.: INT WFS in Virgo, Online Material p 12

Table 2: Continued

\begin{tabular}{|c|c|c|c|c|c|c|c|c|c|c|c|c|c|}
\hline $\begin{array}{l}\text { VCC } \\
\text { (1) }\end{array}$ & $\begin{array}{c}\text { Type } \\
\text { (2) }\end{array}$ & (3) & $\begin{array}{l}m_{p} \\
(4)\end{array}$ & $\begin{array}{c}n \\
(5)\end{array}$ & $\begin{array}{l}\mu_{o} \\
\text { (6) }\end{array}$ & $\begin{array}{l}B_{T} \\
(7)\end{array}$ & $\begin{array}{l}C_{31} \\
(8)\end{array}$ & $\begin{array}{c}<\mu>_{e} \\
\quad \text { (9) }\end{array}$ & $\begin{array}{r}r_{e} \\
(10)\end{array}$ & $\begin{array}{c}\text { Strip } \\
(11)\end{array}$ & $\begin{array}{c}\text { Band } \\
\text { (12) }\end{array}$ & $\begin{array}{c}\text { Notes } \\
\text { (13) }\end{array}$ & $\begin{array}{l}\text { Ref } \\
(14)\end{array}$ \\
\hline 1619 & $\mathrm{E}$ & - & 12.44 & 1.79 & 17.51 & 12.42 & 3.91 & 19.59 & 19.36 & $\mathrm{H}$ & B & 4 & ACS* \\
\hline 1621 & $\mathrm{dE}$ & - & 18.32 & 0.73 & 24.34 & 19.02 & 2.66 & 24.94 & 7.58 & $\mathrm{H}$ & B & & \\
\hline 1626 & $\mathrm{dE}$ & - & 18.95 & 0.85 & 23.88 & 18.84 & 2.60 & 24.79 & 7.48 & $\mathrm{H}$ & B & & \\
\hline 1627 & $\mathrm{E}$ & - & 15.11 & 1.92 & 17.31 & 15.37 & 3.82 & 20.24 & 3.83 & $\mathrm{H}$ & B & & ACS \\
\hline 1630 & $\mathrm{E}$ & - & 12.84 & 1.92 & 17.72 & 12.82 & 4.23 & 20.48 & 15.21 & $\mathrm{H}$ & B & 5 & F97,ACS \\
\hline 1637 & $\mathrm{dE}$ & $\mathrm{N}$ & 18.45 & 1.11 & 23.56 & 18.26 & 3.03 & 24.76 & 8.11 & $\mathrm{H}$ & B & & \\
\hline 1642 & $\mathrm{dE}$ & $\mathrm{N}$ & 17.75 & 1.64 & 23.86 & 17.48 & 5.13 & 25.81 & 19.36 & $\mathrm{H}$ & B & & \\
\hline 1647 & $\mathrm{dE}$ & - & 15.95 & 0.85 & 22.77 & 16.23 & 2.66 & 23.72 & 15.51 & $\mathrm{H}$ & B & & VPC \\
\hline 1663 & $\mathrm{dE}$ & - & 17.45 & 0.71 & 25.22 & 17.33 & 2.35 & 25.86 & 23.32 & $\mathrm{H}$ & B & & \\
\hline 1664 & $\mathrm{E}$ & - & 11.95 & 1.92 & 17.54 & 11.90 & 6.10 & 20.30 & 24.75 & $\mathrm{H}$ & B & 5 & F97,ACS \\
\hline 1689 & $\mathrm{dE}$ & - & 16.95 & 0.79 & 24.23 & 17.46 & 2.51 & 25.07 & 14.62 & $\mathrm{H}$ & B & & \\
\hline 1710 & $\mathrm{dE}$ & - & 17.75 & 0.90 & 22.54 & 17.57 & 2.68 & 23.41 & 6.97 & $\mathrm{H}$ & B & & \\
\hline 1711 & $\mathrm{dE}$ & $\mathrm{N}$ & 16.43 & 0.93 & 22.47 & 16.38 & 2.81 & 23.55 & 11.95 & $\mathrm{H}$ & B & & VPC,BJ98 \\
\hline 1717 & $\mathrm{dE}$ & - & 16.45 & 0.57 & 24.08 & 16.91 & 2.15 & 24.60 & 22.97 & $\mathrm{H}$ & B & & BJ98 \\
\hline 1718 & $\mathrm{dE}$ & $\mathrm{N}$ & 18.25 & 1.05 & 23.96 & 18.48 & 2.86 & 25.13 & 10.67 & $\mathrm{H}$ & B & & \\
\hline 1729 & $\mathrm{dE}$ & - & 17.75 & 0.68 & 24.39 & 18.63 & 2.73 & 24.92 & 10.19 & $\mathrm{H}$ & B & & \\
\hline 1754 & $\mathrm{dE}$ & - & 18.95 & 0.45 & 25.47 & 19.42 & 1.98 & 25.87 & 8.73 & $\mathrm{H}$ & B & & \\
\hline 1783 & $\mathrm{dE}$ & $\mathrm{N}$ & 18.17 & 0.91 & 24.32 & 17.78 & 2.80 & 25.28 & 17.75 & $\mathrm{H}$ & B & & \\
\hline 1785 & $\mathrm{dE}$ & $\mathrm{N}$ & 17.75 & 0.89 & 24.00 & 17.93 & 2.59 & 24.93 & 11.11 & $\mathrm{H}$ & B & & \\
\hline 1794 & $\mathrm{dE}$ & $\mathrm{N}$ & 17.25 & 0.83 & 23.08 & 17.94 & 2.74 & 23.77 & 8.48 & $\mathrm{H}$ & B & & \\
\hline 1803 & $\mathrm{dE}$ & $\mathrm{N}$ & 16.65 & 1.14 & 22.36 & 16.23 & 2.94 & 23.73 & 12.86 & $\mathrm{H}$ & B & & \\
\hline 1812 & $\mathrm{dE}$ & $\mathrm{N}$ & 17.73 & 0.98 & 22.77 & 17.63 & 3.02 & 23.69 & 7.90 & $\mathrm{H}$ & B & & BJ98 \\
\hline 1814 & $\mathrm{dE}$ & - & 18.65 & 0.65 & 23.33 & 20.88 & 2.30 & 23.91 & 1.82 & $\mathrm{H}$ & B & & \\
\hline 1815 & $\mathrm{dE}$ & - & 17.31 & 0.49 & 24.95 & 17.92 & 1.92 & 25.08 & 13.73 & $\mathrm{H}$ & B & & \\
\hline 1831 & $\mathrm{dE}$ & $\mathrm{N}$ & 17.95 & 1.08 & 24.41 & 17.94 & 3.10 & 25.72 & 15.25 & $\mathrm{H}$ & B & & \\
\hline 1861 & $\mathrm{dE}$ & $\mathrm{N}$ & 14.31 & 2.56 & 18.83 & 14.46 & 3.14 & 22.29 & 15.04 & $\mathrm{H}$ & B & 3 & ACS \\
\hline 1863 & $\mathrm{dE}$ & - & 18.94 & 0.54 & 24.51 & 18.75 & 2.15 & 24.87 & 9.07 & $\mathrm{H}$ & B & & \\
\hline 1870 & $\mathrm{dE}$ & - & 15.73 & 2.94 & 19.07 & 16.22 & 3.39 & 22.92 & 14.62 & $\mathrm{H}$ & B & 3 & \\
\hline 1871 & $\mathrm{E}$ & - & 13.80 & 1.61 & 18.31 & 14.43 & 3.41 & 20.67 & 7.17 & $\mathrm{H}$ & B & & ACS \\
\hline 1879 & $\mathrm{dE}$ & $\mathrm{N}$ & 17.25 & 0.95 & 23.78 & 17.32 & 2.79 & 24.90 & 14.64 & $\mathrm{H}$ & B & & \\
\hline 1880 & $\mathrm{dE}$ & - & 18.48 & 0.75 & 24.04 & 18.51 & 2.66 & 24.77 & 8.79 & $\mathrm{H}$ & B & & \\
\hline 1891 & $\mathrm{dE}$ & $\mathrm{N}$ & 16.63 & 1.16 & 22.37 & 17.16 & 3.31 & 23.72 & 10.41 & $\mathrm{H}$ & B & & \\
\hline 1901 & $\mathrm{dE}$ & - & 17.55 & 1.41 & 22.03 & 17.44 & 3.15 & 23.59 & 9.56 & $\mathrm{H}$ & B & & \\
\hline 1903 & $\mathrm{E}$ & - & 10.70 & 3.03 & 15.80 & 10.88 & 5.53 & 20.60 & 39.47 & $\mathrm{H}$ & B & 1,5 & F97,ACS \\
\hline 1904 & $\mathrm{dE}$ & $\mathrm{N}$ & 18.95 & 0.60 & 26.17 & 17.72 & 2.66 & 26.50 & 22.86 & $\mathrm{H}$ & B & & \\
\hline 1909 & $\mathrm{dE}$ & $\mathrm{N}$ & 16.05 & 1.04 & 22.35 & 16.50 & 3.22 & 23.39 & 12.78 & $\mathrm{H}$ & B & & \\
\hline 1910 & $\mathrm{dE}$ & $\mathrm{N}$ & 14.12 & 1.47 & 19.79 & 14.41 & 3.13 & 21.86 & 13.22 & $\mathrm{H}$ & B & & ACS \\
\hline 1915 & $\mathrm{dE}$ & - & 17.08 & 0.68 & 23.89 & 17.20 & 2.65 & 24.42 & 14.58 & $\mathrm{H}$ & B & & BJ98 \\
\hline 1942 & $\mathrm{dE}$ & $\mathrm{N}$ & 16.72 & 0.79 & 23.03 & 16.81 & 2.85 & 23.96 & 13.33 & $\mathrm{H}$ & B & & BJ98 \\
\hline 1945 & $\mathrm{dE}$ & $\mathrm{N}$ & 14.77 & 1.01 & 22.00 & 15.23 & 3.48 & 23.15 & 21.61 & $\mathrm{H}$ & B & & \\
\hline 1951 & $\mathrm{dE}$ & $\mathrm{N}$ & 16.95 & 1.16 & 23.66 & 16.92 & 3.14 & 24.91 & 16.10 & $\mathrm{H}$ & B & & \\
\hline 1958 & $\mathrm{dE}$ & $\mathrm{N}$ & 16.93 & 0.92 & 22.57 & 16.88 & 2.86 & 23.52 & 9.45 & $\mathrm{H}$ & B & & \\
\hline 1971 & $\mathrm{dE}$ & - & 16.53 & 1.19 & 22.02 & 16.72 & 3.24 & 23.35 & 10.78 & $\mathrm{H}$ & B & & \\
\hline 1982 & $\mathrm{dE}$ & - & 15.24 & 0.87 & 21.84 & 16.02 & 2.72 & 22.84 & 13.67 & $\mathrm{H}$ & B & 1 & \\
\hline 1986 & $\mathrm{dE}$ & $\mathrm{N}$ & 18.95 & 1.35 & 24.68 & 18.16 & 3.55 & 26.11 & 16.00 & $\mathrm{H}$ & B & & \\
\hline 1991 & $\mathrm{dE}$ & $\mathrm{N}$ & 15.55 & 1.12 & 22.54 & 15.66 & 2.91 & 23.93 & 20.13 & $\mathrm{H}$ & B & & \\
\hline 1995 & $\mathrm{dE}$ & - & 15.75 & 1.05 & 22.64 & 15.90 & 2.81 & 23.95 & 18.18 & $\mathrm{H}$ & B & & \\
\hline 2000 & $\mathrm{E}$ & - & 11.87 & 2.17 & 16.03 & 12.11 & 4.84 & 19.46 & 13.56 & $\mathrm{H}$ & B & 3,5 & ACS* \\
\hline 2001 & $\mathrm{dE}$ & - & 18.95 & 0.79 & 24.26 & 18.02 & 2.54 & 25.16 & 11.46 & $\mathrm{H}$ & B & & \\
\hline 2003 & $\mathrm{dE}$ & - & 18.15 & 0.96 & 24.33 & 17.98 & 3.06 & 25.34 & 15.50 & $\mathrm{H}$ & B & & \\
\hline 2008 & $\mathrm{dE}$ & - & 14.95 & 0.86 & 23.16 & 15.06 & 2.72 & 24.21 & 40.74 & $\mathrm{H}$ & B & & \\
\hline 2010 & $\mathrm{dE}$ & - & 18.95 & 0.69 & 24.42 & 18.88 & 2.39 & 25.07 & 8.35 & $\mathrm{H}$ & B & & \\
\hline 2011 & $\mathrm{dE}$ & - & 17.05 & 0.72 & 24.00 & 17.40 & 2.61 & 24.65 & 13.05 & $\mathrm{H}$ & B & & \\
\hline 2012 & $\mathrm{dE}$ & $\mathrm{N}$ & 14.22 & 1.30 & 21.77 & 14.74 & 2.97 & 23.30 & 22.42 & $\mathrm{H}$ & B & 1 & \\
\hline 2025 & $\mathrm{dE}$ & - & 18.45 & 1.32 & 23.80 & 18.34 & 3.83 & 25.62 & 13.80 & $\mathrm{H}$ & B & & \\
\hline 2032 & $\mathrm{dE}$ & - & 17.45 & 1.08 & 24.60 & 17.18 & 2.95 & 25.91 & 24.12 & $\mathrm{H}$ & B & & \\
\hline 2049 & $\mathrm{dE}$ & $\mathrm{N}$ & 16.35 & 0.65 & 22.72 & 16.52 & 2.38 & 23.26 & 15.19 & $\mathrm{H}$ & B & 1 & \\
\hline 2050 & $\mathrm{dE}$ & $\mathrm{N}$ & 15.13 & 1.12 & 21.05 & 15.23 & 3.03 & 22.98 & 16.37 & $\mathrm{H}$ & B & & ACS \\
\hline 2051 & $\mathrm{dE}$ & - & 17.45 & 0.78 & 22.91 & 17.24 & 2.65 & 23.75 & 9.29 & $\mathrm{H}$ & B & & \\
\hline 2056 & $\mathrm{dE}$ & - & 16.95 & 1.15 & 21.53 & 16.43 & 3.18 & 22.98 & 9.59 & $\mathrm{H}$ & B & & \\
\hline 2072 & $\mathrm{dE}$ & - & 18.95 & 0.47 & 25.10 & 19.54 & 1.76 & 25.53 & 7.31 & $\mathrm{H}$ & B & & \\
\hline 2078 & $\mathrm{dE}$ & - & 17.45 & 1.15 & 24.10 & 16.26 & 3.24 & 25.49 & 32.61 & $\mathrm{H}$ & B & & \\
\hline 2081 & $\mathrm{dE}$ & - & 16.95 & 0.82 & 23.59 & 17.11 & 2.70 & 24.47 & 13.82 & $\mathrm{H}$ & B & & \\
\hline
\end{tabular}

Column 1: VCC designation.

Column 2,3: Morphological type from the VCC. N=Nucleated.

Column 4: Photographic magnitude $m_{p}$ from the VCC.

Column 5: Sersic index $n$.

Column 6: Sersic extrapolation to $r=0$ of the central surface brightness $\mu_{o}$ (in mag $\operatorname{arcsec}^{-2}$ ).

Column 7: Total asymptotic magnitude $B_{T}$.

Column 8: Light concentration index $C_{31}$.

Column 9: Mean effective surface brightness $\langle\mu\rangle_{e}$ (in mag $\operatorname{arcsec}^{-2}$ ).

Column 10: Effective major-axis radius $r_{e}$ (in $\operatorname{arcsec}$ ).

Column 11: WFS strip: $\mathrm{H}=$ horizontal; $\mathrm{V}=$ =vertical.

Column 12: Available band(s).

Column 13: Notes. $(1=$ poor outer fit; 2 = M32 like object; 3 = two seeing disks excluded from the fit; 4 = core; $5=$ power law; $6=$ saturated.

Column 14: Reference to other works. B03 = Barazza et al (2003), F97 = Faber et al. (1997), VPC = Young \& Currie (1998), BJ98 = Binggeli \& Jerijn 1998; ACS = in The ACS Virgo Cluster Survey,

$\mathrm{ASC}^{*}=$ whether core or power law, kindly provided by Laura Ferrarese (private communication). 